\title{
Maternally expressed 3 protects the intestinal barrier from cardiac arrest-induced ischemia/reperfusion injury via miR-34a-3p/sirtuin 1/nuclear factor kappa B signaling
}

\author{
Xianwei Huang", Mandong Pan", Penghui Du, Yinrong Chen, Caixia Zhang, Wang Lu, Jiyan Lin \\ Emergency Department, The First Affiliated Hospital of Xiamen University, Xiamen, China \\ Contributions: (I) Conception and design: X Huang; (II) Administrative support: J Lin; (III) Provision of study materials or patients: M Pan; (IV) \\ Collection and assembly of data: P Du; (V) Data analysis and interpretation: Y Chen; (VI) Manuscript writing: All authors; (VII) Final approval of \\ manuscript: All authors. \\ \#These authors contributed equally to this work. \\ Correspondence to: Jiyan Lin, MD. Emergency Department, The First Affiliated Hospital of Xiamen University, No.55, Zhenhai Road, Xiamen \\ 361003, China. Email: happylinjiy@163.com.
}

Background: Cardiac arrest (CA), a common disease with a high mortality rate, is a leading cause of ischemia/reperfusion (I/R)-induced dysfunction of the intestinal barrier. Long non-coding RNAs (lncRNAs) play crucial roles in multiple pathological processes. However, the effect of the lncRNA maternally expressed 3 (MEG3) on intestinal I/R injury and the intestinal barrier has not been fully determined. Therefore, this study aimed to investigate the function of MEG3 in CA-induced intestinal barrier dysfunction.

Methods: The oxygen and glucose deprivation (OGD) model in the human colorectal adenocarcinoma Caco-2 cells and in vivo cardiac arrest-induced intestinal barrier dysfunction model in Sprague-Dawley (SD) rats were established. The effect and underlying mechanism of MEG3 on the intestinal barrier from cardiac arrest-induced ischemia/reperfusion injury were analyzed by methyl thiazolyl tetrazolium (MTT) assays, Annexin V-FITC/PI apoptosis detection kit, Terminal deoxynucleotidyl transferase-mediated dUTP nick end labelling (TUNEL) staining, quantitative polymerase chain reaction (qPCR) assays, Western blot analysis, luciferase reporter gene assays, transepithelial electrical resistance (TEER) measurements, immunofluorescence analysis, and enzyme-linked immunosorbent assay (ELISA) assays.

Results: Interestingly, we found that MEG3 could protect Caco-2 cells from oxygen-glucose deprivation (OGD)/reoxygenation-induced I/R injury by modulating cell proliferation and apoptosis. Moreover, MEG3 relieved OGD-induced intestinal barrier dysfunction in vitro, as demonstrated by its significant rescue effect on transepithelial electrical resistance and the expression of tight junction proteins such as occludin and claudin-1 (CLDN1), which were impaired in OGD-treated Caco-2 cells. Mechanistically, MEG3 inhibited the expression of inflammatory factors including interleukin (IL)-1 $\beta$, tumor necrosis factor (TNF)- $\alpha$, interferon-gamma (IFN)- $\gamma$, inflammatory factors including interleukin (IL)-10, and transforming growth factor beta (TGFb)-1, as well as nuclear factor-kappa B (NF- $\kappa$ B) signaling. In response to OGD treatment in vitro, MEG3 also activated the expression of sirtuin 1 (SIRT1) by Caco-2 cells via sponging miR-34a-3p. Furthermore, MEG3 relieved CA-induced intestinal barrier dysfunction through NF- $\mathrm{B}$ signaling in vivo.

Conclusions: LncRNA MEG3 can protect the intestinal barrier from cardiac arrest-induced I/R injury via miR-34a-3p/SIRT1/NF- $\mathrm{B}$ signaling. This finding provides new insight into the mechanism by which MEG3 restores intestinal barrier function following I/R injury, presenting it as a potential therapeutic candidate or strategy in intestinal injury.

Keywords: Cardiac arrest (CA); intestinal barrier; lncRNA MEG3; miR-34a-3p; nuclear factor kappa B (NF-кB); sirtuin1 (SIRT1) 
Submitted Aug 13, 2020. Accepted for publication Dec 26, 2020.

doi: $10.21037 /$ atm-20-6438

View this article at: http://dx.doi.org/10.21037/atm-20-6438

\section{Introduction}

Cardiac arrest (CA) is a common cause of mortality in patients with coronary aorta disorder (1). CA sees the heart rate rise dramatically, and the contractions of cardiac muscle in the ventricular cells become uncoordinated; this causes the heart to pump inefficiently, resulting in unconsciousness and death $(2,3)$. CA-induced microvascular dysfunction, insufficient blood flow, and tissue oxygen transportation lead to systemic ischemia/reperfusion (I/R) injury in various organs, including the brain and intestine (4). Pathophysiological intestinal $\mathrm{I} / \mathrm{R}$ can also result from acute mesenteric ischemia, surgical procedure, and traumatic shock $(5,6)$. Intestinal $\mathrm{I} / \mathrm{R}$ can give rise to numerous organ disorders and inflammatory system syndromes and is a major cause of mortality and morbidity in surgical patients $(7,8)$.

After CA, reestablishing the blood flow and oxygen supply are vital in heart and intestine. The reperfusion triggers a cascade of events that may inflict further injury and usually surpasses the initial ischemic damage $(9,10)$. Intestinal I/R destroys the function of the mucosal barrier $(11,12)$. By enhancing the permeability of the intestinal barrier, intestinal I/ $\mathrm{R}$ induces the translocation of endotoxins and pathogenic bacteria, which eventually progresses to multiple organ failure and sepsis $(13,14)$. Therefore, the intestinal mucosal barrier, which is responsible for defending the body from invasion by pathogenic organisms, should be protected from I/R injury caused by CA (15). However, the mechanism underlying CA-induced intestinal I/R injury and intestinal mucosal barrier dysfunction has not been fully elucidated.

The advances in high-throughput technology have facilitated a profound exploration of the non-coding genome with unparalleled determination. Long non-coding RNAs (lncRNAs) have been found to play crucial roles in multiple physiological and pathological processes (16). LncRNAs have also been observed to impact mRNA paracellular permeability and translation, as well as intestinal cell proliferation and apoptosis susceptibility, to regulate intestinal I/R and the intestinal barrier (17). A recent study reported that lncRNA uc. 173 regulates the intestinal barrier by modulating miR-29b/claudin-1 (CLDN1) signaling (18). Maternally expressed gene 3
(MEG3) is an imprinted gene located at chromosome12 in mice and chromosome 14 in humans which expresses in many normal tissues, such as cancer cells, neurons, hepatocytes, cardiac fibroblasts, and ECs. MEG3 is a well-known lncRNA that plays various roles in multiple pathological processes, including cancer progression and inflammatory response (18-20). Many reports reveal that MEG3 could inhibit tumor cell proliferation and induces tumor cell apoptosis as well as autophagy. Those researches suggest that MEG3 exerts an antitumor activity in several cancers. The involvement of lncRNA MEG3 in neurological dysfunction has also been demonstrated in a brain I/R injury mouse model (21). It has been also reported MEG3 can regulate IL-1 $\beta$ abundance to prevent sepsis during lung infection by acting as a decoy of miRNA (22). Piccoli et al. identifies the lncRNA Meg3 to be a crucial regulator of cardiac MMP-2, promoting cardiac fibrosis and impairment of diastolic function following pressure overload (23). However, the effect of MEG3 on intestinal $\mathrm{I} / \mathrm{R}$ injury and the intestinal mucosal barrier remains unclear.

MicroRNAs (miRNAs), a type of small RNAs that modulate mRNA translation, regulate the expression of genes involving in various biological and medical processes, such as including cell cycle regulation, stress response, differentiation, inflammation, and cancer progression $(24,25)$. Several miRNAs, including miR-381-3p, miR$130 \mathrm{a}$, and miR-212, have been reported to participate in the modulation of intestinal I/R injury and the intestinal barrier by targeting various key signaling pathways $(26,27)$. MiR$34 a-3 p$ plays a vital role in the development of malignancies including cervical cancer and meningioma $(28,29)$.

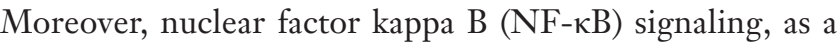
critical pathway for multiple cellular processes, contributes to intestinal I/R injury and intestinal barrier dysregulation (30-32). Sirtuin 1 (SIRT1), as a highly conserved NAD+dependent deacetylases and a critical biological sensor, has been well recognized to inhibit NF- $\mathrm{\kappa B}$ signaling in multiple pathological processes, and the miRNA targeting SIRT1 can induce NF- $\kappa \mathrm{B}$ activation (33-36). Nevertheless, the relationship between miR-34a-3p, SIRT1/NF- $\kappa$ B signaling, and MEG3, especially in the modulation of intestinal I/R and the intestinal barrier, remains obscure.

In this study, we aimed to explore the role of MEG3 
in CA-induced intestinal $\mathrm{I} / \mathrm{R}$ and intestinal barrier dysregulation. We identified a novel protective role of MEG3 against CA-induced intestinal I/R injury in the intestinal barrier via miR-34a-3p/SIRT1/NF- $\kappa$ B signaling.

We present the following article in accordance with the ARRIVE reporting checklist (available at http://dx.doi. org/10.21037/atm-20-6438).

\section{Methods}

\section{Cell culture, treatment and oxygen-glucose deprivation (OGD) model}

Human colorectal adenocarcinoma Caco-2 cells were supplied by American Type Tissue Culture Collection (New York, USA). The cells were cultured in Dulbecco's Modified Eagle Medium (DMEM, Solarbio, China) containing 10\% fetal bovine serum (Gibco, USA), $0.1 \mathrm{mg} / \mathrm{mL}$ streptomycin and 100 units $/ \mathrm{mL}$ penicillin at $37{ }^{\circ} \mathrm{C}$ with $5 \% \mathrm{CO}_{2}$. To simulate I/R conditions, an in vitro OGD model was established. Caco-2 cells were incubated for $12 \mathrm{~h}$ with $5 \%$ $\mathrm{CO}_{2}$ and $1 \% \mathrm{O}_{2}$ with $94 \% \mathrm{~N} 2$. The cells were then cultured for $6 \mathrm{~h}$ in normoxic conditions to achieve reoxygenation. The cells were infected with ADV4, ADV4 MEG3, ADV1, and ADV1 MEG3 shRNA. All vectors were synthesized by Genscript (Nanjing, China). The MEG3 shRNA target sequence was: 5'-GCUCAUACUUUGACUCUAU-3'. MiR-34a-3p mimic and inhibitor were obtained from RiboBio (Guangzhou, China). Pyrrolidinedithiocarbamate (PDTC) (Sigma, USA) was used at a dose of $60 \mathrm{mM}$.

\section{MTT assays}

MTT assays were performed to measure the cell viability of Caco- 2 cells. Briefly, $2 \times 10^{4}$ Caco- 2 cells were put into 96-well plate and cultured for $12 \mathrm{~h}$. Then, $10 \mu \mathrm{L}$ MTT solution $(5 \mathrm{mg} / \mathrm{mL})$ was added, and the cells were cultured for a further $4 \mathrm{~h}$. The medium was discarded, and $150 \mu \mathrm{L}$ dimethyl sulfoxide (DMSO) was added to the wells. An enzyme-linked immunosorbent assay (ELISA) microplate reader was used to analyze the absorbance at $570 \mathrm{~nm}$ (BioTek EL 800, USA).

\section{Analysis of cell apoptosis}

Caco-2 cells were cultured at a density of $1 \times 10^{5}$ in 6-well culture plates. Cell apoptosis was measured using an Annexin V-FITC/PI apoptosis detection kit (Keygen
Biot Solasodine, China) according to the manufacturer's instructions. Briefly, $1 \times 10^{6}$ cells were collected and washed by binding buffer. The cells were then dyed with Annexin $\mathrm{V}$ and propidium iodide (PI) at $25^{\circ} \mathrm{C}$. Finally, apoptotic cells were measured by flow cytometry.

\section{Terminal deoxynucleotidyl transferase-mediated dUTP nick end labelling (TUNEL) staining}

Apoptotic cells were also detected by TUNEL staining kit supplied by Ruisai Biotechnology Co., Ltd. (Shanghai, China) following the manufacturer's manual. Five random fields were selected in each section and the rate of positive cells was analyzed under a fluorescence microscope at $400 x$ magnification.

\section{Quantitative reverse transcription-polymerase chain reaction ( $q R T-P C R)$}

Total RNAs was extracted using TRIzol (Invitrogen, USA). Synthesis of first-strand cDNA was carried out according to the manufacturer's instructions (Invitrogen, USA). Quantitative reverse transcription-polymerase chain reaction (qRT-PCR) was conducted using SYBR Premix Ex Taq II kit (Takara, Japan). The standard controls for miRNA and mRNA were U6 and glyceraldehyde 3-phosphate dehydrogenase (GAPDH), respectively. The quantitative RNA levels were determined in three independent experiments. The primer sequences used were as follows: MEG3 forward: 5'-GTGAAGGTCGGAGTGAACG-3', reverse: 5'-CTCGCTCCTGGAAGATGGTG-3'; miR-34a-3p forward: 5'-ACACTCCAGCTGGG AATCAGCAAGTATACTGCCCTA-3', reverse: 5 '-CTCAACTGGTGTCGTGGAGTCGGCAAT TCAGTTGAGTAGGGCAG-3'; SIRT1 forward: 5'-CTTCAGGTCAAGGGATGGTAT-3', reverse: 5'-GCGTGTCTATGTTCTGGGTAT-3'; U6 forward: 5'-GACAGATTCGGTCTGTGGCAC-3', reverse: 5'-GATTACCCGTCGGCCATCGATC-3'; GAPDH forward: 5'-GACAGATTCGGTCTGTGGCAC-3', reverse: 5'-GATTACCCGTCGGCCATCGATC-3'.

\section{Western blot analysis}

Caco-2 cells were collected with RIPA (Beyotime, China) with phenylmethylsulfonyl fluoride (PMSF). Identical amounts of protein were divided by sodium dodecyl sulphate-polyacrylamide gel electrophoresis (SDS- 
PAGE), and then transferred to polyvinylidene difluoride (PVDF) membranes (Millipore, USA). The membranes were blocked with $5 \%$ milk and incubated with primary antibodies for B-cell lymphoma 2 (Bcl-2, Proteintech, China), Bcl-2-associated X protein (Bax, Proteintech, China), cyclin D1 (Proteintech, China), SIRT1 (Proteintech, China), caspase-3 (Cell Signaling, US), p-p65 (Bioworld Technology, USA), p65 (Bioworld Technology, USA), p-ІкB (Bioworld Technology, USA), IкB (Bioworld Technology, USA), and GAPDH (Sigma, USA) at $4{ }^{\circ} \mathrm{C}$ overnight. Then, the membranes were exposed to the corresponding secondary antibodies for $1 \mathrm{~h}$. Finally, the proteins were visualized using a Bio-Rad imaging system.

\section{Luciferase reporter gene assay}

Luciferase reporter gene assays were carried out using the Dual-luciferase Reporter Assay System (Promega, USA). Briefly, samples were transfected with miR-1247-3p mimic, miR-control, and the vector containing MEG3, MEG3 mutant, SIRT1, or SIRT1 mutant fragment using Lipofectamine 2000 (Invitrogen, USA). Luciferase activity was analyzed and Renilla was used for normalization.

\section{Transepithelial electrical resistance (TEER) measurements}

To analyze the barrier function of the intestine, TEER was measured. $2 \times 10^{5}$ cells $/ \mathrm{mL}$ Caco- 2 cells were layered on collagen-covered polycarbonate penetrable support supplements (Corning, USA). The medium was replaced every 2 days. The intestinal barrier was measured at 3 weeks by TEER using the Millicell ERS-2 (Electrical Resistance System; Merck-Millipore, USA).

\section{Immunofluorescence analysis}

Caco-2 cells were fixed by $4 \%$ paraformaldehyde for $30 \mathrm{~min}$, treated with Triton $\mathrm{X} 100(0.2 \%)$ for $10 \mathrm{~min}$, and then treated with bovine serum albumin (BSA, 2\%) for 30 minutes. The slides were incubated with occludin (Proteintech, China) and CLDN1 (Proteintech, China) antibodies at $4{ }^{\circ} \mathrm{C}$ overnight, and then incubated with secondary antibodies (Proteintech, China) for $1 \mathrm{~h}$ at $37^{\circ} \mathrm{C}$. The slides were stained with Hoechst (Beyotime, China) for $10 \mathrm{~min}$ at $25^{\circ} \mathrm{C}$. A Nikon microscope (Tokyo, Japan) was used to analyze immunofluorescence.

\section{ELISA assays}

The levels of interleukin (IL)-1 $\beta$, tumor necrosis factor (TNF)- $\alpha$, interferon-gamma (IFN)- $\gamma$, IL-10, and transforming growth factor beta (TGFb)-1 in the culture medium of Caco-2 cells were analyzed with a doubleantibody ELISA kit (R\&D, USA).

\section{Establishment of a CA rat model}

Sprague-Dawley (SD) rats were used to establish a CAinduced intestinal barrier dysfunction model in vivo, as previously described (37). Briefly, two acupuncture pins were placed in the fourth rib of the right sternal edge and the left sternal edge. The stimulating wire was attached, and a $60-\mathrm{Hz}, 2 \mathrm{~mA}$ current was delivered. The current was maintained for 3 minutes to avoid unintentional defibrillation. After 6 minutes, the resuscitation was started; the rats were given adrenaline $(2.2 \mathrm{~g} / 100 \mathrm{~g})$ administration, chest compression (200 times/min), and mechanical ventilation $\left(100 \% \mathrm{FiO}_{2}, 0.65 \mathrm{~mL} / 100 \mathrm{~g}\right.$, 100 breaths/min). The mechanical oxygenation and hemodynamic monitoring were maintained with $21 \%$ oxygen for a further $2 \mathrm{~h}$. After recovery, the rats were closely monitored.

Next, the rats were intravenously injected via the tail with ADV4, ADV4 MEG3, ADV1, ADV1 MEG3 shRNA or treated with percutaneous tunneled drainage catheter (PTDC) for further analysis. The serum levels of D-lactic acid and endotoxin in the rats were measured using the corresponding kit (Horseshoe Crab, China). Sections of the intestinal mucosa of the rats were stained with hematoxylin and eosin (HE). Immunohistochemical (IHC) staining with the appropriate antibodies (Proteintech, China) was also performed to analyze the expression levels of occludin and CLDN1 in the intestinal mucosa of the rats. The animal care and experimental procedures were authorized by the Animal Ethics Committee of The First Affiliated Hospital of Xiamen University (20190227), in compliance with the First Affiliated Hospital of Xiamen University guidelines for the care and use of animals.

\section{Statistical analysis}

Data were expressed as mean $\pm \mathrm{SD}$, and the statistical analysis was performed by GraphPad prism 7. The unpaired Student's 
$t$-test was applied for comparing two groups, and the oneway ANOVA was applied for comparing among multiple groups. $\mathrm{P}<0.05$ were considered as statistically significant.

\section{Results}

\section{LncRNA MEG3 protected Caco-2 cells from OGD-induced I/R injury in vitro}

Cell proliferation and apoptosis have been reported to be the crucial contributors to intestinal I/R injury (38). To assess the effect of lncRNA MEG3 on intestinal I/R injury in vitro, we constructed an OGD model in the human colorectal adenocarcinoma Caco-2 cells by treating them with $\mathrm{OGD} /$ reoxygenation (OGD/R) to mimic intestinal I/R injury.

Interestingly, we found that the expression levels of MEG3 were reduced in the OGD cell model, in which the overexpression of MEG3 could rescue this reduction whereas the depletion of MEG3 by shRNA could further decrease the expression in the system (Figure 1A), suggesting that OGD-induced intestinal I/R hindered the expression of MEG3 in Caco-2 cells. As expected, cell viability was decreased in OGD-treated Caco-2 cells (Figure $1 B$ ). Surprisingly, MEG3 overexpression increased the cell viability, but the MEG3 shRNA decreased cell viability in the cells, indicating that MEG3 enhances cell proliferation in response to OGD-induced cell injury (Figure $1 B$ ). Consistently, cell apoptosis was attenuated by MEG3 overexpression, while the knockdown of MEG3 enhanced cell apoptosis in the OGD-treated cells (Figure 1C,D).

Several key proteins, including Bcl-2, Bax, and caspase-3, are involved in the modulation of cell apoptosis and can serve as markers of apoptosis $(39,40)$. Intriguingly, we found that MEG3 overexpression rescued the expression of Bcl-2 in OGD-treated cells and reduced the expression of Bax and caspase- 3 induced by OGD treatment, while the depletion of MEG3 exerted the opposite effect (Figure 1E). These results suggested that MEG3 can protect Caco-2 cells from OGD-induced I/R injury by modulating apoptosis.

\section{LncRNA MEG3 relieved OGD-induced intestinal barrier dysfunction in vitro}

Next, we investigated the role of MEG3 in the regulation of the intestinal barrier in vitro. TEER is an indicator of intestinal barrier function (41). Interestingly, the TEER value in Caco-2 cells was significantly reduced after OGD treatment (Figure 2A). Strikingly, the OGD-inhibited TEER level was rescued by the overexpression of MEG3 and further decreased by the MEG3 shRNA (Figure 2A), suggesting that MEG3 can alleviate the impairment of the intestinal barrier by OGD. Besides, as a control, TEER values in the monolayers were not changed (Figure 2B). Tight junction proteins including occludin and CLDN1, play a critical role in maintaining the function of the intestinal barrier (42). Significantly, immunofluorescence revealed that the expression levels of occludin and CLDN1 were reduced in OGD-treated Caco-2 cells; this effect could be rescued by MEG3 overexpression but was reinforced by the depletion of MEG3 (Figure 2C,D), indicating that MEG3 relieved OGD-induced intestinal barrier dysfunction in vitro.

\section{$N F-\kappa B$ signaling might be involved in the protective mechanism of MEG3 against OGD-induced I/R injury in vitro}

Next, we attempted to determine the mechanism underlying MEG3's mediation of the intestinal barrier in OGD-induced I/R injury. Surprisingly, we found that the expression levels of inflammatory factors including IL- $1 \beta$, TNF- $\alpha$, IFN- $\gamma$, IL-10, and TGFb-1 in the culture medium of OGD-treated Caco-2 cells were significantly attenuated by MEG3 overexpression, whereas cells transfected with MEG3 shRNA enhanced the expression levels of IL-1 $\beta$, TNF- $\alpha$, IFN- $\gamma$, IL-10, and TGFb-1 (Figure $3 A, B, C, D, E$ ). These observations suggested that MEG3 protects against inflammation in OGD-related cell injury. The phosphorylation of $\mathrm{p} 65$ subunits of NF- $\mathrm{\kappa B}$ has been reported to be necessary for p65 activation (43). Moreover, the

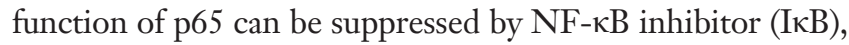
with the phosphorylation of $\mathrm{I} \kappa \mathrm{B}$ causing I $\kappa \mathrm{B}$ degradation and the activation of p65 (44). We found that the levels of p65 phosphorylation (p-p65) and p65 downstream gene cyclin D1 were up-regulated, whereas phosphorylation of

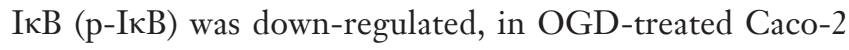
cells (Figure 3F). Remarkably, these effects could be rescued by MEG3 overexpression and enhanced by the depletion of MEG3 (Figure 3F), suggesting that MEG3 impaired NF$\kappa \mathrm{B}$ signaling in the OGD model. Moreover, the NF- $\kappa \mathrm{B}$ inhibitor PDTC could reverse the effect of MEG3 depletion on cell proliferation and apoptosis as demonstrated by the increases in cell viability and $\mathrm{Bcl}-2$ expression, as well as the decreased expression of Bax (Figure 3G,H). Taken 
A

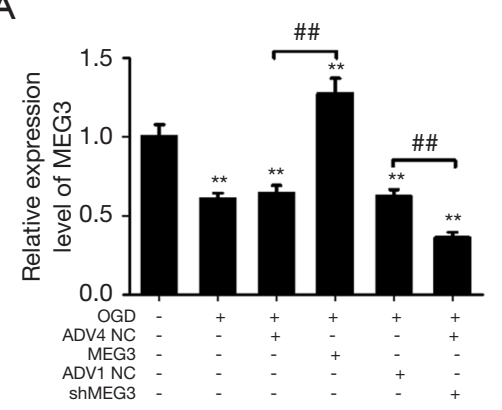

C

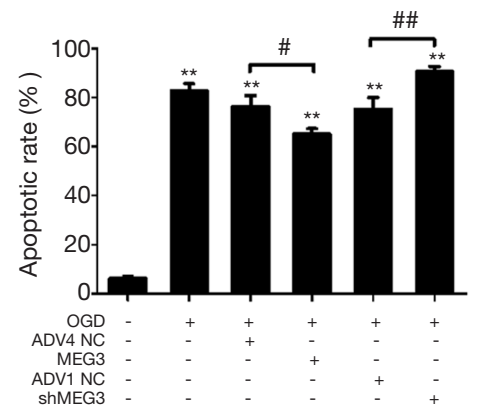

B

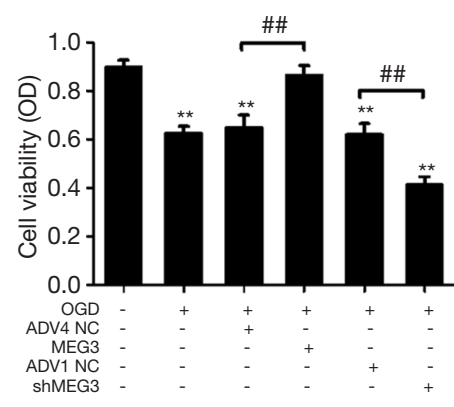

Control

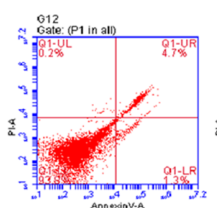

OGD

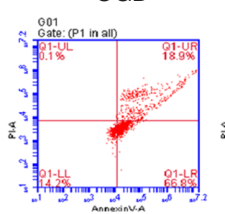

OGD+ADV4 NC

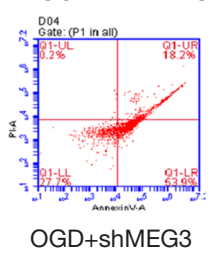

OGD+MEG3
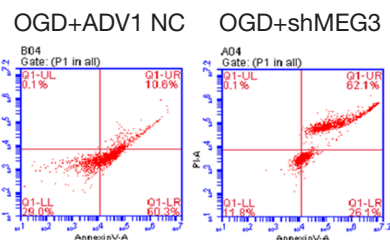

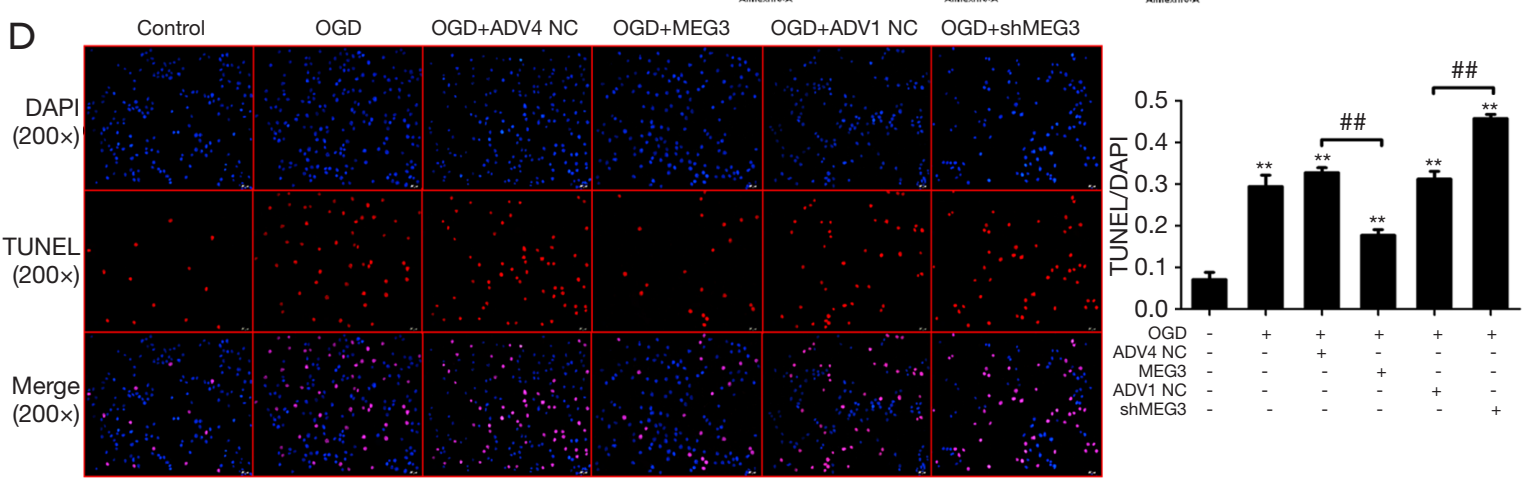

$\mathrm{E}$

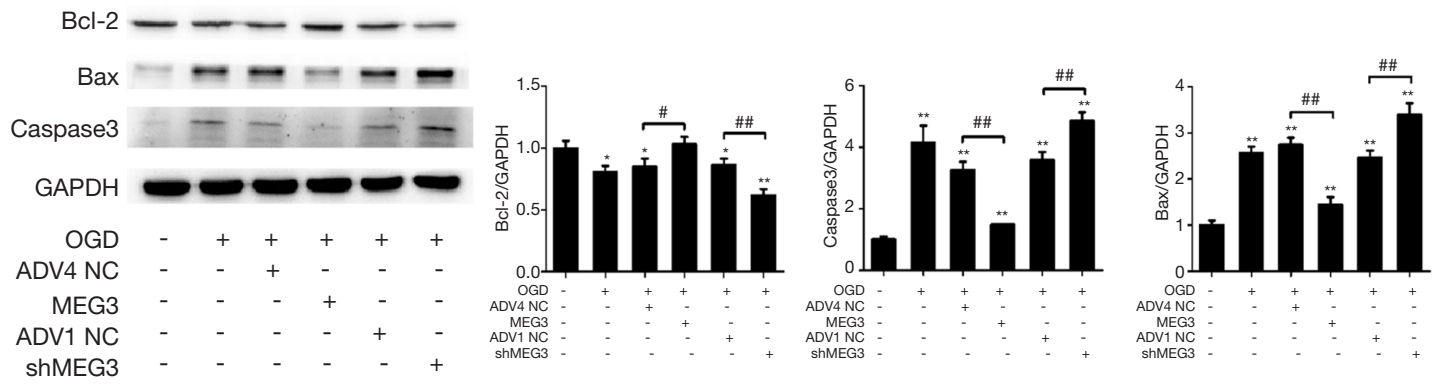

Figure 1 MEG3 protects Caco-2 cells from OGD induced I/R injury in vitro. (A-E) Untreated Caco-2 cells and oxygen and glucose deprivation-treated Caco-2 cells were infected with ADV4, ADV4 MEG3, ADV1, or ADV1 MEG3 shRNA as indicated. (A) The expression levels of lncRNA MEG3 were measured by qPCR. (B) Cell viability was analyzed by MTT assays. (C) Cell apoptosis was assessed by flow cytometry. (D) Cell apoptosis was assessed by TUNEL staining. Mean \pm SD of at least three experiments is shown. (E) The expression levels of Bcl-2, Bax, caspase-3, and GAPDH were detected by western blot. Statistically significant differences are indicated: ${ }^{*} \mathrm{P}<0.01,{ }^{\#} \mathrm{P}<0.05$,

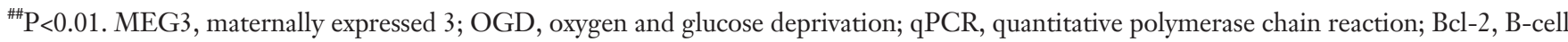
lymphoma 2; Bax, B cell lymphoma/leukmia-2 (Bcl-2) associated x protein; GAPDH, glyceraldehyde-3-phosphate dehydrogenase; SD, standard deviation; I/R, ischemia/reperfusion. 
A

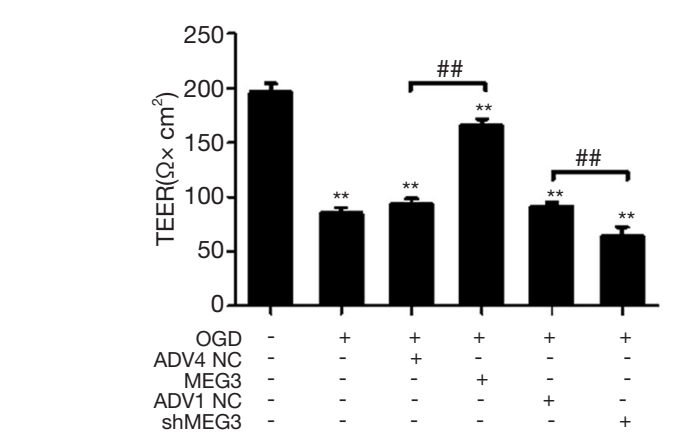

B
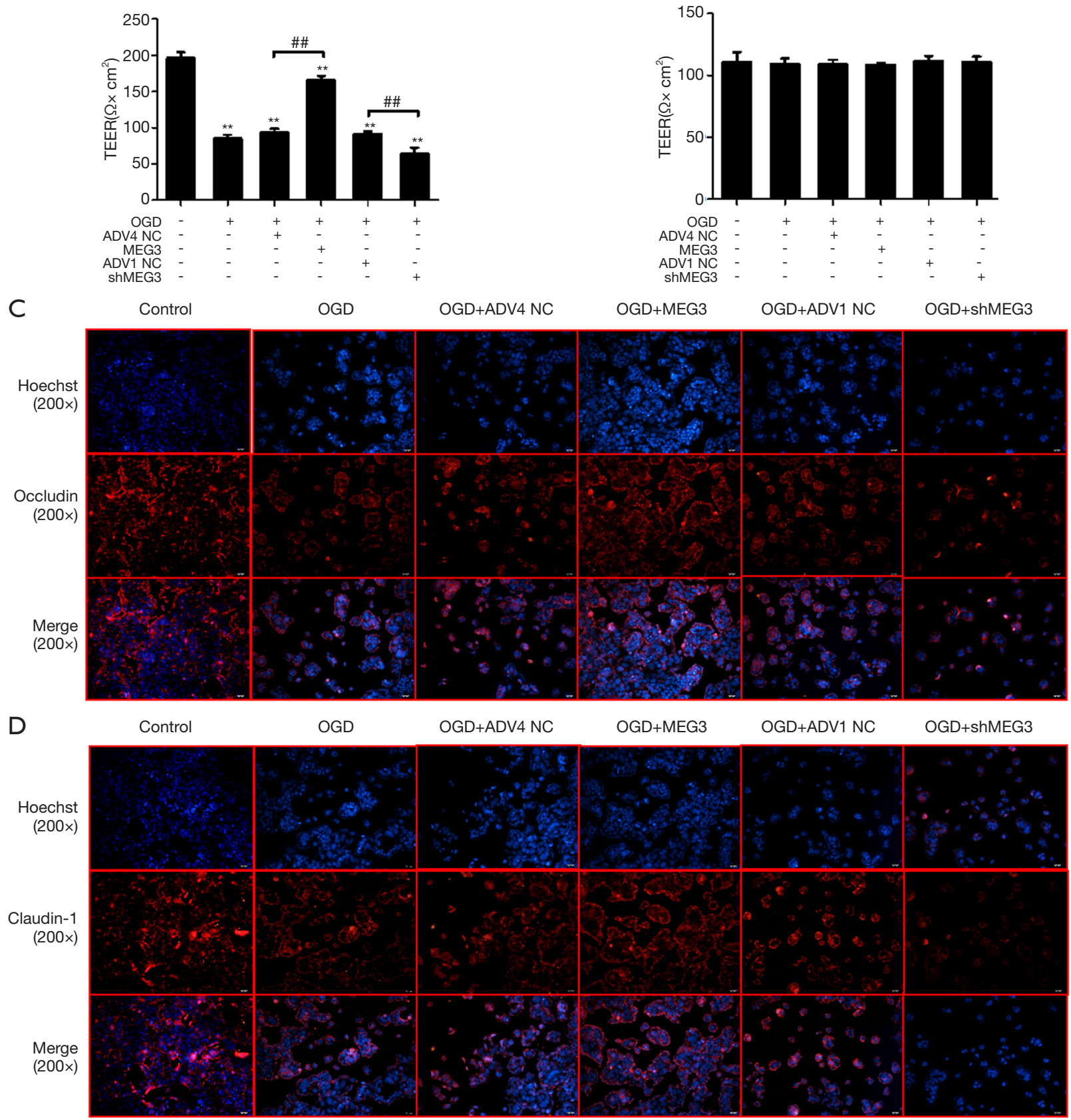

Figure 2 MEG3 relieves OGD-induced intestinal barrier dysfunction in vitro. (A,B) Untreated Caco-2 cells and oxygen and glucose deprivation-treated Caco-2 cells were infected with ADV4, ADV4 MEG3, ADV1, or ADV1 MEG3 shRNA as indicated. (A,B) The transepithelial electrical resistance (TEER) levels were measured, with the TEER values in the monolayers serving as a control; (C,D) the expression levels of occludin and claudin-1 were measured by immunofluorescence analysis. Mean \pm SD of at least three experiments is shown. Statistically significant differences are indicated: ${ }^{* *} \mathrm{P}<0.01,{ }^{\# \#} \mathrm{P}<0.01$. SD, standard deviation; OGD, oxygen and glucose deprivation. 

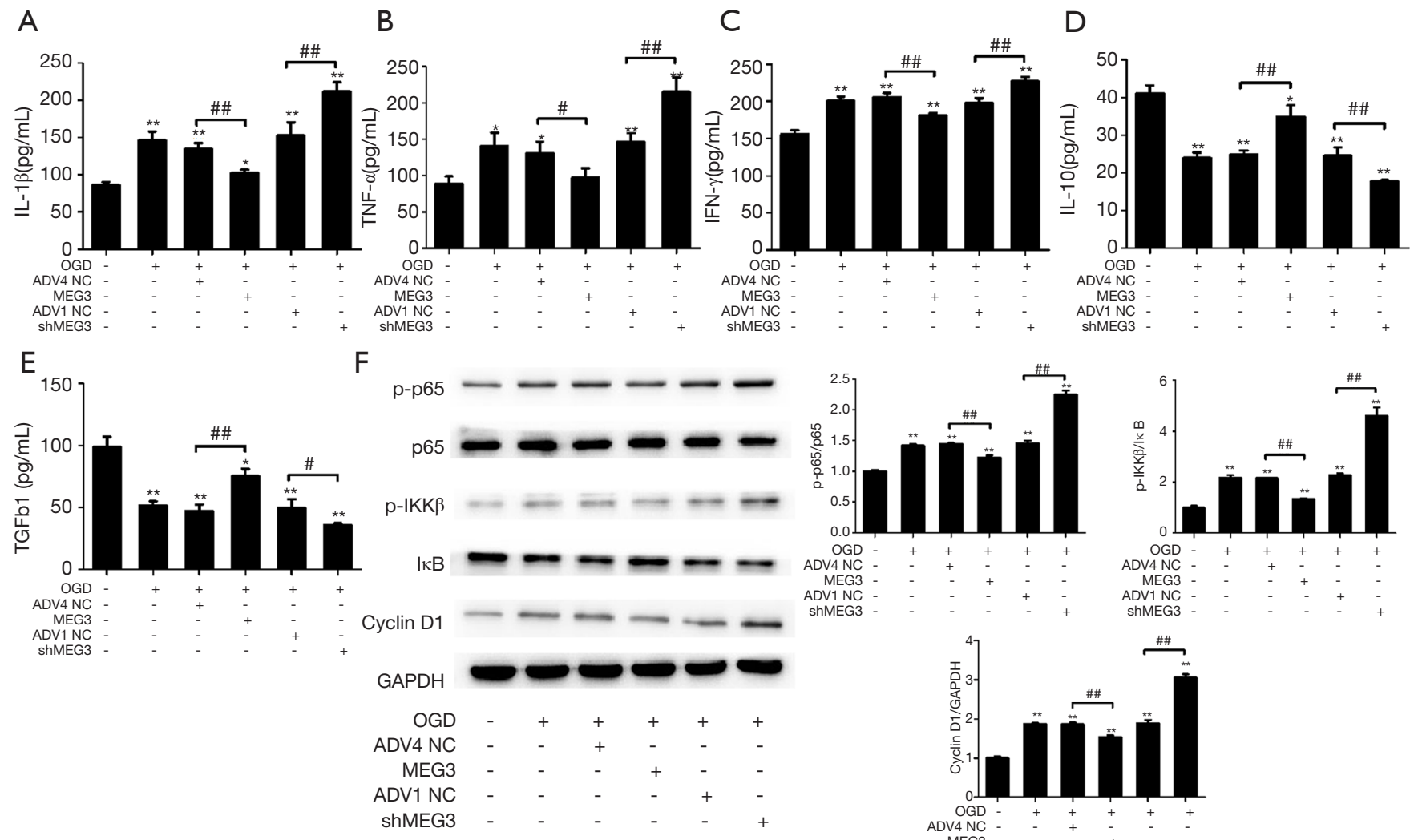

G

$\mathrm{H}$
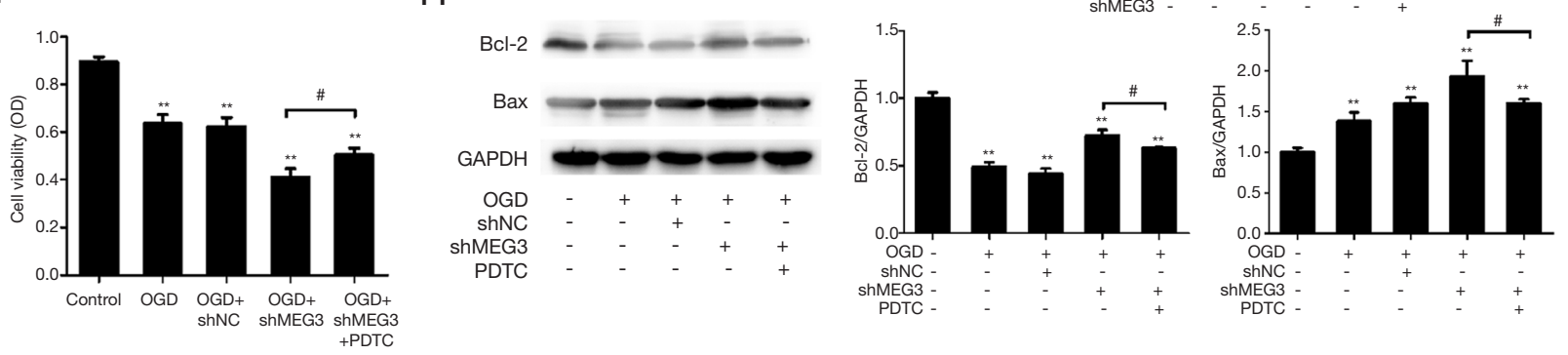

Figure $3 \mathrm{NF}-\kappa \mathrm{B}$ signaling is involved in the protective effect of MEG3 against OGD-induced Caco-2 cell I/R injury. (A-F) Untreated Caco-2 cells and OGD-treated Caco-2 cells were infected with ADV4, ADV4 MEG3, ADV1, or ADV1 MEG3 shRNA as indicated. (A-E) The levels of IL-1 $\beta$, TNF- $\alpha$, IFN- $\gamma$, IL-10, and TGFb-1 in the culture medium of the cells were determined by ELISA assays. (F) The

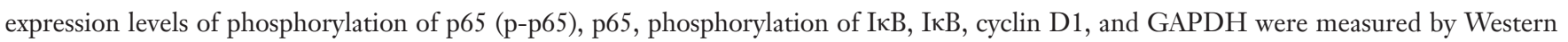
blot analysis. $(\mathrm{G}, \mathrm{H})$ Untreated Caco-2 cells or OGD-treated Caco-2 cells were infected with ADV1, ADV1 MEG3 shRNA or treated with PDTC as indicated. (G) Cell viability was analyzed by MTT assays. (H) The expression levels of Bcl-2, Bax, and GAPDH were detected by western blot. Mean $\pm \mathrm{SD}$ of at least three experiments is shown. Statistically significant differences are indicated: ${ }^{*} \mathrm{P}<0.05,{ }^{* *} \mathrm{P}<0.01,{ }^{*} \mathrm{P}<0.05$, ${ }^{\#} \mathrm{P}<0.01$. IL-1 $\beta$, interleukin-1 $\beta$; TNF- $\alpha$, tumor necrosis factor- $\alpha$; IFN- $\gamma$, interferon-gamma- $\gamma$; IL-10, inflammatory factors including interleukin -10; TGFb-1, transforming growth factor beta-1; ELISA, enzyme-linked immunosorbent assay; NF- $\kappa \mathrm{B}$, nuclear factor-kappa B; MEG3, maternally expressed 3; OGD, oxygen and glucose deprivation; I/R, ischemia/reperfusion. 
together, these findings indicated that NF- $\mathrm{BB}$ signaling may be involved in MEG3-induced protective function against OGD-induced I/R injury in vitro.

\section{MEG3 attenuated OGD-induced I/R injury in vitro by sponging miR-34a-3p}

Next, we further investigated the mechanism by which MEG3 modulates the activation of NF- $\mathrm{B}$ signaling. MiRNAs have been reported to be involved in lncRNAmediated NF- $\kappa \mathrm{B}$ signaling $(45,46)$. Therefore, we hypothesized that miRNA was involved in MEG3regulated p65 inactivation. We performed a bioinformatics analysis using the DIANA tools web server (http://carolina. imis.athena-innovation.gr/diana_tools/web/index.php). Interestingly, we identified that MEG3 contained a binding sequence of miR-34a-3p (Figure $4 A$ ). Furthermore, we observed that miR-34a-3p mimic significantly reduced the luciferase activity of wild-type MEG3 but did not affect that of MEG3 with mutation of the miR-34a-3p binding site (Figure 4B), which indicated that MEG3 can was able to target miR-34a-3p. Meanwhile, OGD treatment enhanced the expression of miR-34a-3p in Caco-2 cells in which this effect was impaired by MEG3 overexpression and enhanced by MEG3 depletion (Figure 4C). Remarkably, the inhibition of MEG3 by OGD treatment was enhanced by miR-34a-3p mimic, whereas miR-34a-3p inhibitor rescued MEG3 expression (Figure 4D), which indicated that MEG3 responds to OGD treatment in vitro by sponging miR-34a$3 p$. Moreover, miR-34a-3p mimic inhibited the cell viability of OGD-treated cells, while miR-34a-3p inhibitor enhanced the cell viability (Figure 4E). More importantly, in OGDtreated Caco-2 cells miR-34a-3p mimic impaired MEG3 overexpression-induced cell proliferation and reversed MEG3 overexpression-inhibited cell apoptosis (Figure 4F,G,H). These results suggested that MEG3 attenuated OGDinduced I/R injury in vitro by sponging miR-34a-3p.

\section{MEG3 activated SIRT1 expression in response to OGD treatment in vitro by sponging miR-34a-3p}

Next, we further explored the potential target of miR$34 a-3 p$ in the MEG3-mediated NF- $\mathrm{B}$ signaling pathway. SIRT1 has been shown to inhibit NF- $\mathrm{KB}$ signaling, and miRNA can target SIRT1, thereby activating p65 function $(36,47,48)$. We performed a bioinformatics analysis using Targetscan (http://www.targetscan.org/vert_72/) to identify the miR-34a-3p-targeted site within SIRT1 3' untranslated region (UTR) (Figure 5A). Luciferase reporter gene assays showed that the luciferase activity of wild-type SIRT1 but not that of SIRT1 with mutation of the miR-34a-3p binding site was reduced by miR-34a-3p mimic (Figure 5B), indicating that miR-34a-3p can target SIRT1. The expression levels of SIRT1 were reduced by OGD treatment and further inhibited by miR-34a-3p mimic but enhanced by miR-34a$3 \mathrm{p}$ inhibitor (Figure $5 \mathrm{C}$ ); these observations suggest that miR-34a-3p is able to down-regulate SIRT1. Moreover, the overexpression of MEG3 significantly rescued OGDdecreased SIRT1 expression, while miR-34a-3p mimic impaired this rescue (Figure 5D). These findings suggested that MEG3 activated the expression of SIRT1 in response to OGD treatment in vitro by sponging miR-34a-3p.

\section{MEG3 protected Caco-2 cells from OGD-induced I/R injury in vitro via miR-34a-3p/NF-кB signaling}

We further explored the function of MEG3/miR-34a-3p/ $\mathrm{NF}-\kappa \mathrm{B}$ signaling in OGD-induced I/R injury in Caco2 cells. Interestingly, we found that the NF- $\kappa \mathrm{B}$ inhibitor PDTC rescued the decreased expression of MEG3 and blocked the expression of miR-34a-3p induced by OCD in a feedback event (Figure 6A,B). Significantly, PDTC attenuated the upregulation of IL- $1 \beta$ and TNF- $\alpha$ levels caused by MEG3 depletion and further consolidated the inhibitive effect of MEG3 overexpression on IL-1 $\beta$, TNF- $\alpha$, IFN- $\gamma$, IL-10, and TGFb-1 expression (Figure 6C,D,E,F, G). Similar results were observed in the western blot analysis of p-p65, p-IкB, and cyclin D1 (Figure 6H). Moreover, PDTC attenuated the MEG3 overexpression-inhibited on cell apoptosis and MEG3 depletion-induced cell apoptosis as demonstrated by increased $\mathrm{Bcl}-2$ expression and decreased Bax expression (Figure 6I). Together, our findings suggested that MEG3 protected Caco-2 cells from OGD-induced I/R injury via miR-34a-3p/NF- $\mathrm{kB}$ signaling in vitro.

\section{MEG3 relieved CA-induced intestinal barrier dysfunction via $N F-\kappa B$ signaling in vivo}

Next, we examined the role of MEG3 in the CA-induced intestinal I/R injury and intestinal barrier dysfunction in vivo by establishing a CA-induced I/R rat model. We found that the levels of lactic acid and endotoxin in the serum of the CA-treated rats were elevated. Moreover, these increases were blocked by MEG3 overexpression and reinforced by MEG3 depletion (Figure $7 A, B$ ). Meanwhile, PDTC attenuated the increases in the levels of lactic 
A

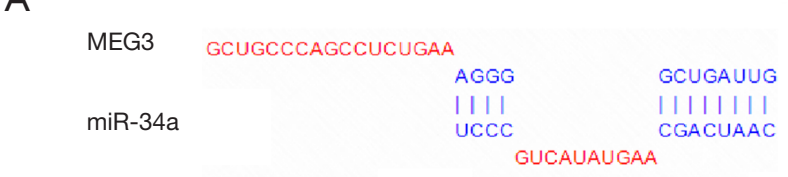

$\mathrm{B}$

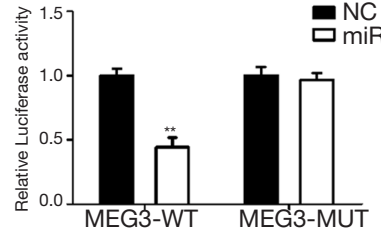

C

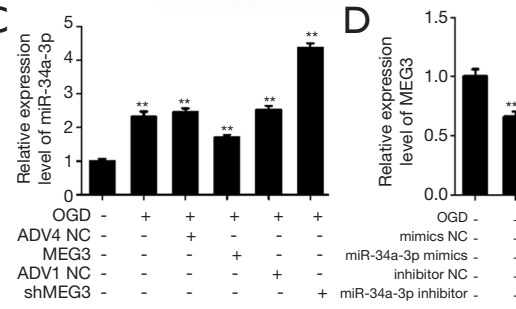

G

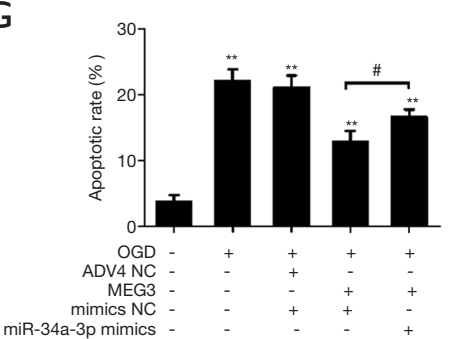

Control OGD

E

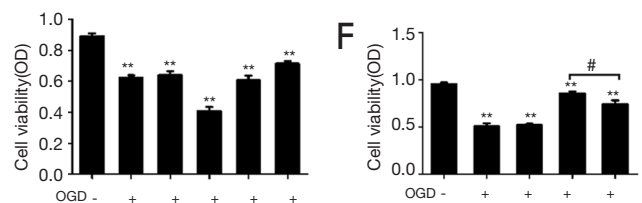

mimics NC -

inhibitor NC

inhibitor NC
+ miR-34a-3p inhibitor

OGD+ADV4 NC+mimics NC
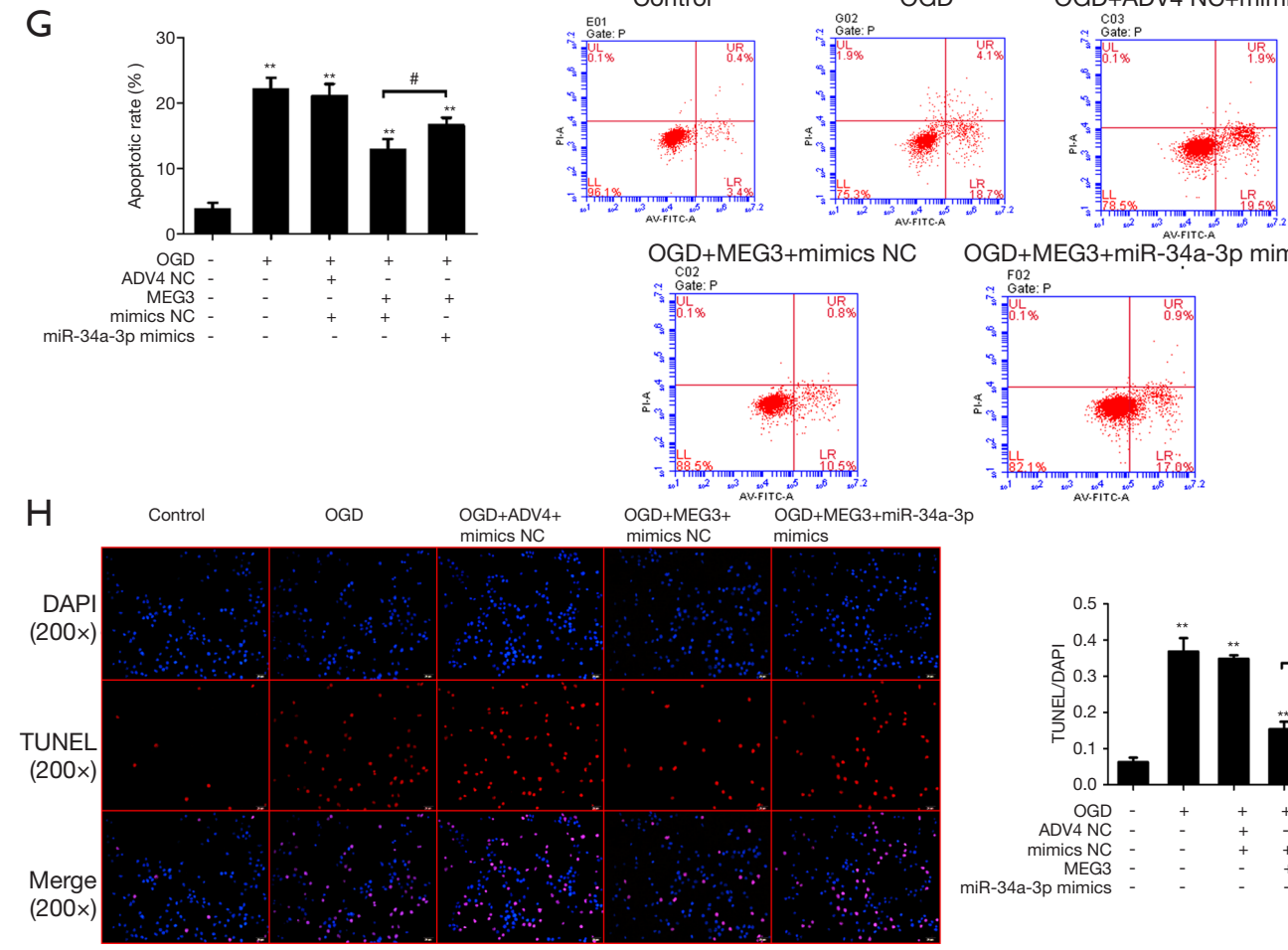

OGD+MEG3+mimics NC OGD+MEG3+miR-34a-3p mimics
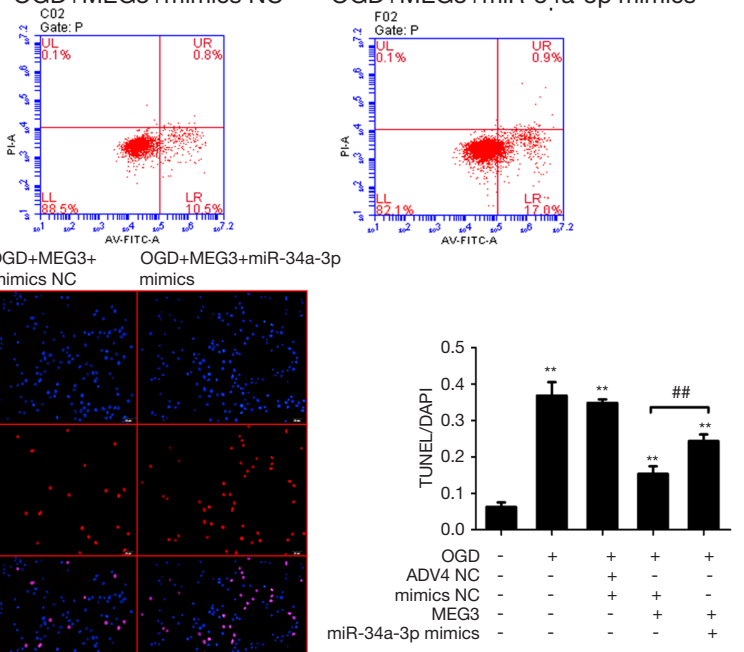

Figure 4 MEG3 sponges miR-34a-3p to protect against OGD-induced Caco-2 cell I/R injury. (A) The interaction of MEG3 and miR34a-3p was identified by bioinformatics analysis using the DIANA tools web-server (http://carolina.imis.athena-innovation.gr/diana_tools/ web/index.php). (B) The luciferase activities of wild-type MEG3 (MEG3-WT) and MEG3 with mutation of the miR-34a-3p binding site (MEG3-MUT) were analyzed by luciferase reporter gene assays in Caco-2 cells treated with the control or miR-34a-3p mimic. (C) Caco-2 cells and OGD-treated Caco-2 cells were infected with ADV4, ADV4 MEG3, ADV1 or ADV1 MEG3 shRNA, as indicated. The expression levels of miR-34a-3p were measured by qPCR. (D,E) Caco-2 cells and OGD-treated Caco-2 cells were treated with control, miR-34a-3p mimic, or miR-34a-3p inhibitor, as indicated. (D) The expression levels of MEG3 were determined by qPCR. (E) Cell viability was analyzed by MTT assays. (F,G) Caco-2 cells and OGD-treated Caco-2 cells were infected with ADV4, ADV4 MEG3, control, or miR-34a-3p mimic, as indicated. (F) Cell viability was analyzed by MTT assays. (G) Cell apoptosis was assessed by flow cytometry. (H) Cell apoptosis was assessed by TUNEL staining. Mean $\pm \mathrm{SD}$ of at least three experiments is shown. Statistically significant differences are indicated: ${ }^{*} \mathrm{P}<0.05$, ${ }^{* *} \mathrm{P}<0.01,{ }^{\#} \mathrm{P}<0.05,{ }^{\# \#} \mathrm{P}<0.01$. MEG3, maternally expressed 3; OGD, oxygen and glucose deprivation; qPCR, quantitative polymerase chain reaction; SD, standard deviation; I/R, ischemia/reperfusion; TUNEL, Terminal deoxynucleotidyl transferase-mediated dUTP nick end labelling. 
A

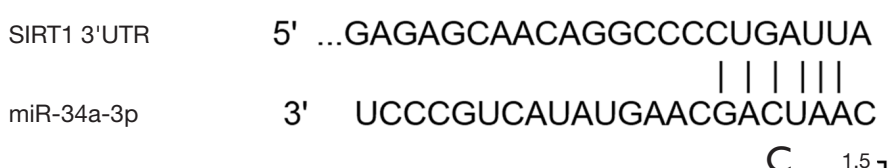

B

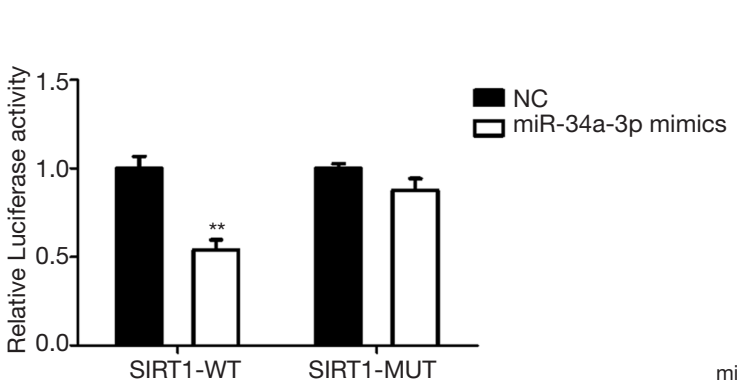

C $\quad 1.5$
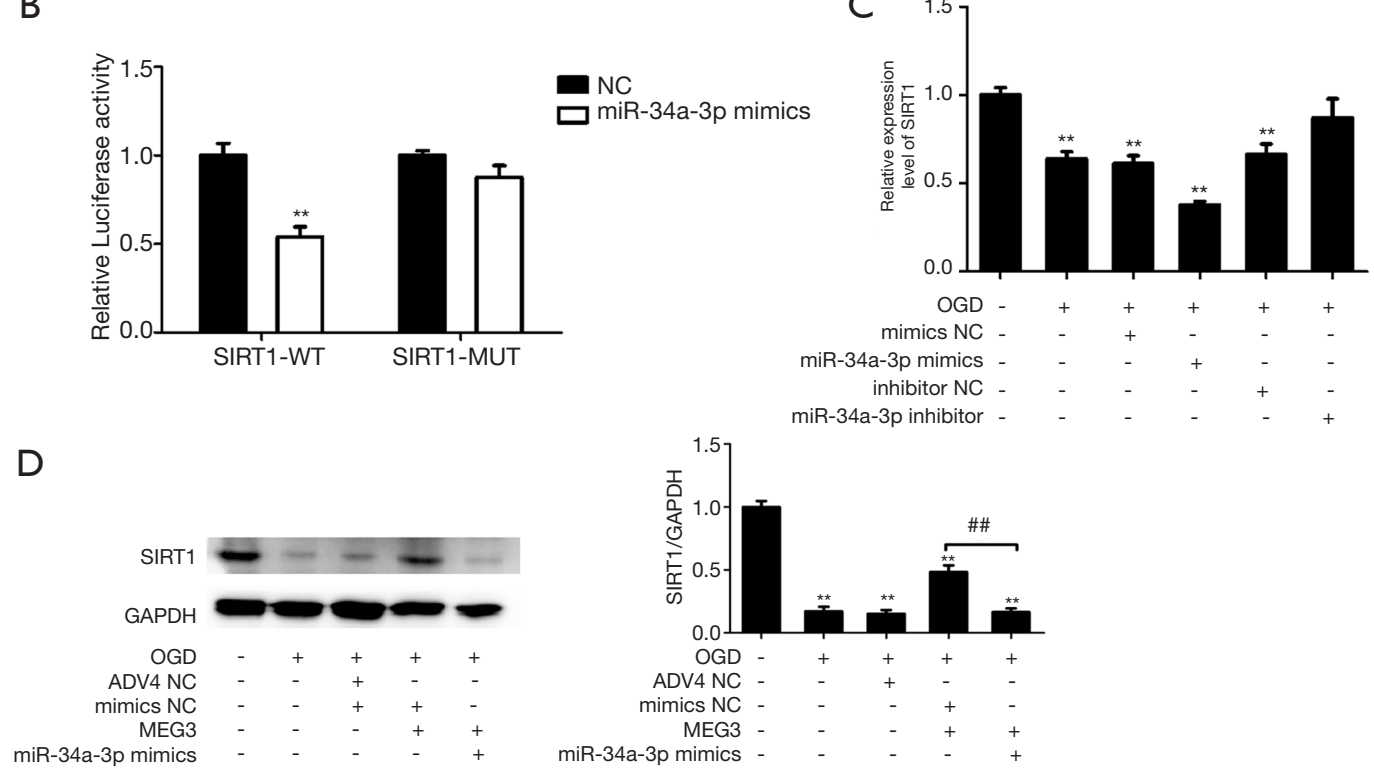

Figure 5 MEG3 activates the expression of SIRT1 by sponging miR-34a-3p in response to OGD treatment in vitro. (A) The interaction between SIRT1 and miR-34a-3p was identified by bioinformatics analysis using Targetscan (http://www.targetscan.org/vert_72/) (B) The luciferase activity of wild-type SIRT1 SIRT1-WT) and SIRT1 with mutation of the miR-34a-3p binding site (SIRT1-MUT) were analyzed by luciferase reporter gene assays of Caco-2 cells treated with control or miR-34a-3p mimic. (C) Caco-2 cells or OGD-treated Caco-2 cells were treated with control, miR-34a-3p mimic, or miR-34a-3p inhibitor, as indicated. The expression levels of SIRT1 were analyzed by qPCR. (D) Caco-2 cells or OGD-treated Caco-2 cells were infected with ADV4, ADV4 MEG3, control, or miR-34a-3p mimic as indicated. The expression levels of SIRT1 were measured by western blot. Mean \pm SD of at least three experiments is shown. Statistically significant differences are indicated: ${ }^{* *} \mathrm{P}<0.01,{ }^{\# \#} \mathrm{P}<0.01$. MEG3, maternally expressed 3; OGD, oxygen and glucose deprivation; qPCR, quantitative polymerase chain reaction; SD, standard deviation; SIRT1, sirtuin 1.

acid and endotoxin induced by MEG3 depletion but enhanced the decreases in these levels caused by MEG3 overexpression (Figure $7 A, B$ ). Similar results were observed by HE staining of sections of intestinal mucosa from the rats (Figure $7 C$ ). Furthermore, the expression levels of occludin and claudin-1 in the intestinal mucosa of the rats were significantly reduced by CA-induced intestinal I/R, and this effect could be rescued by MEG3 overexpression but reinforced by the depletion of MEG3 (Figure 7D,E). Additionally, PDTC reversed the occludin and CLDN1 expression caused decreased by MEG3 depletion but further enhanced the MEG3 overexpression-elevated expression of these genes (Figure 7A,B). Taken together, our results suggested that MEG3 alleviated CA-induced barrier

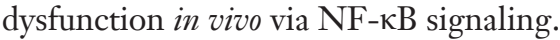

\section{Discussion}

Intestinal I/R causes intestinal barrier dysfunction through various mechanisms (49). Nurr1 has been reported to support the restoration of the intestine after I/R injury via repressing p21 (50). Phosphatase and tensin homolog (PTEN) perform an essential role in relieving renal dysfunction after I/R by preserving the capacity of the intestinal barrier (51). MitoQ defends the function of the intestinal barrier by alleviating mitochondrial DNA damage via the Nrf2/ARE pathway (52). Ginsenoside $\mathrm{Rb} 1$ was shown to inhibit intestinal I/R-induced oxidative 
A

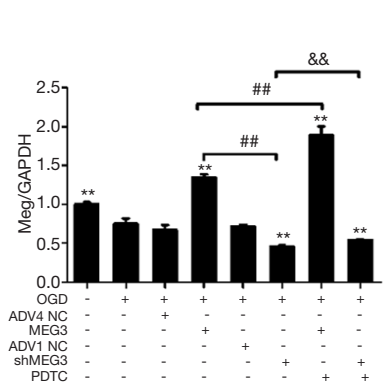

$\mathrm{E}$

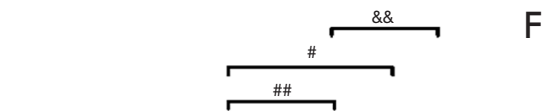

B

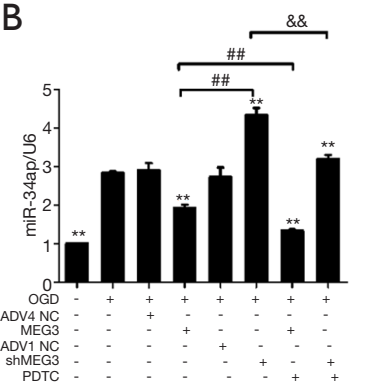

$\mathrm{F}$
C

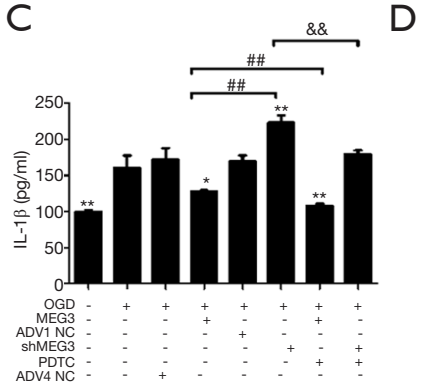

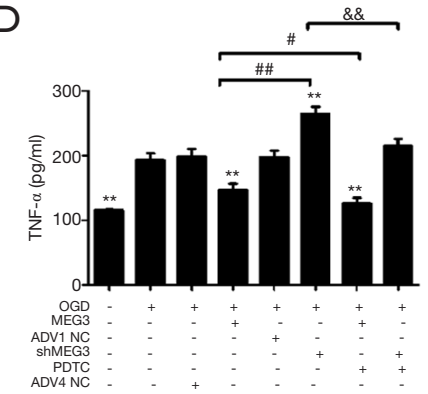

G
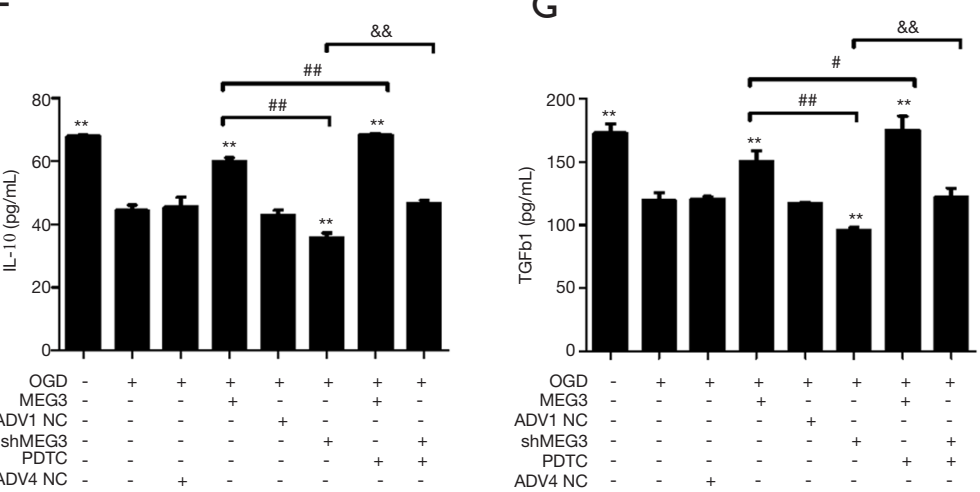

$\mathrm{H}$
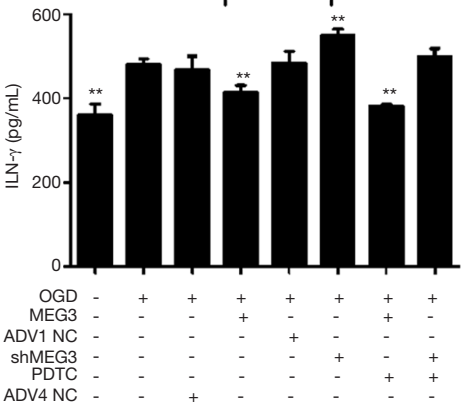

ADV4 NC
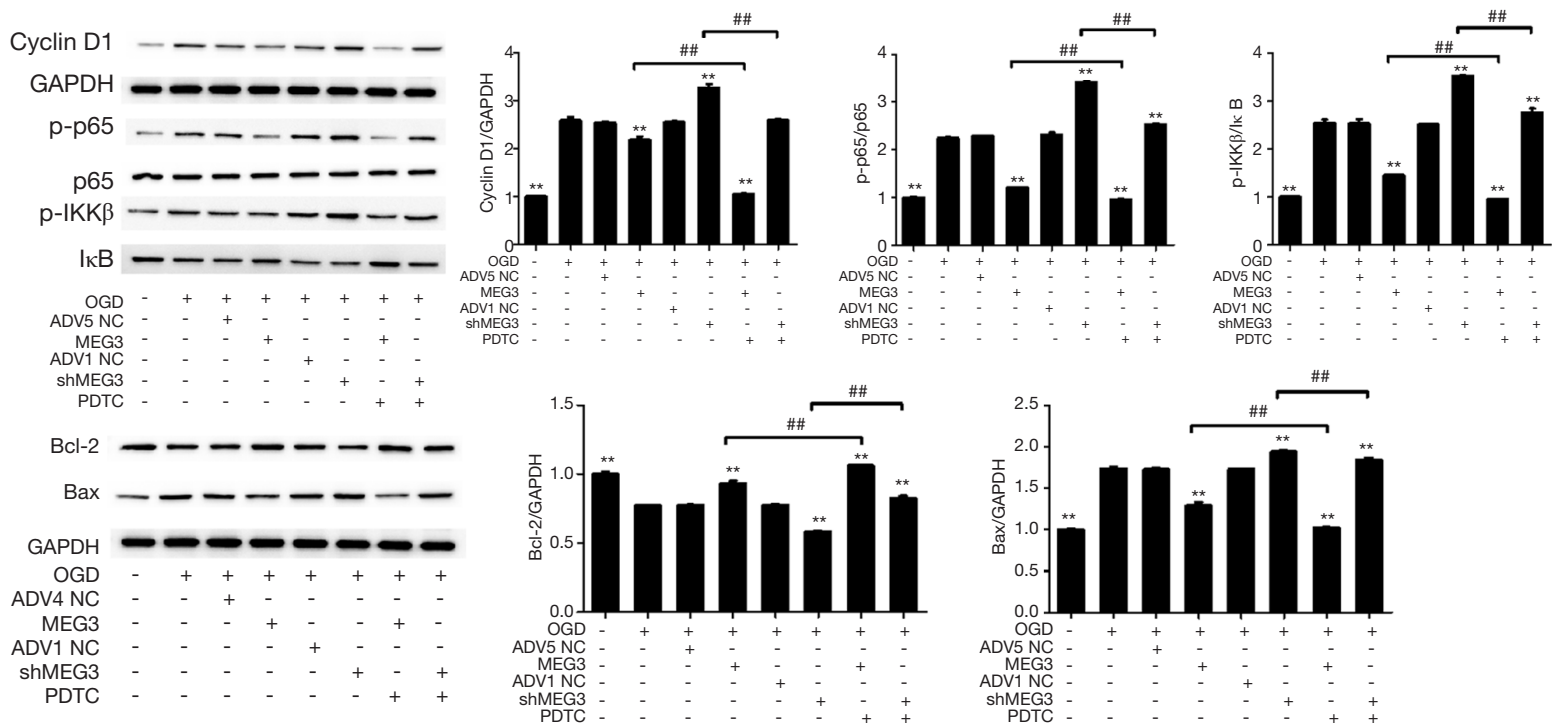

Figure 6 MEG3 protects Caco-2 cells from OGD-induced I/R injury via miR-34a-3p/NF-кB signaling. (A-I) Untreated or OGD-treated Caco-2 cells were infected with ADV4, ADV4 MEG3, ADV1, or ADV1 MEG3 shRNA or treated with PDTC as indicated. (A) The expression levels of MEG3 were analyzed by qPCR. (B) The expression levels of miR-34a-3p were measured by qPCR. (C-G) The levels of IL-1 $\beta$, TNF- $\alpha$, IFN- $\gamma$, IL-10, and TGFb-1 in the culture medium of the cells were determined by ELISA assays. (H) The expression levels of phosphorylation of p65 (p-p65), p65, phosphorylation of IкB, IкB, cyclin D1, and GAPDH were assessed by western blot. (I) The expression levels of Bcl-2, Bax, and GAPDH were detected by western blot. Mean $\pm \mathrm{SD}$ of at least three experiments is shown. Statistically significant differences are indicated: ${ }^{*} \mathrm{P}<0.05,{ }^{* *} \mathrm{P}<0.01,{ }^{\#} \mathrm{P}<0.05,{ }^{\#} \mathrm{P}<0.01,{ }^{*}{ }^{*} \mathrm{P}<0.01$. NF- $\mathrm{kB}$, nuclear factor-kappa $\mathrm{B} ;$ MEG3, maternally expressed 3; OGD, oxygen and glucose deprivation; I/R, ischemia/reperfusion; IL-1 $\beta$, interleukin-1 $\beta$; TNF- $\alpha$, tumor necrosis factor- $\alpha$; IFN- $\gamma$, interferon-gamma- $\gamma$; IL-10, inflammatory factors including interleukin 10; TGFb-1, transforming growth factor beta-1; ELISA, enzyme-linked immunosorbent assay; SD, standard deviation. 
A

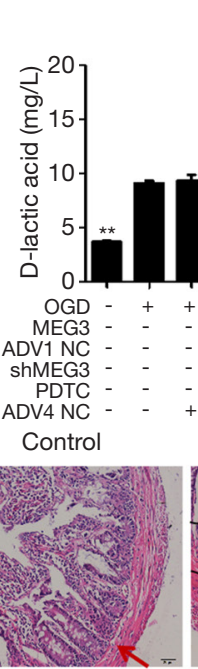

OGD+ShMEG3
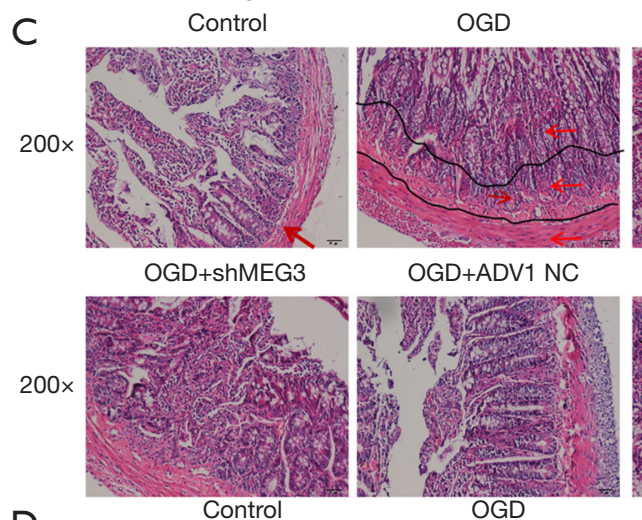

D

$\mathrm{E}$

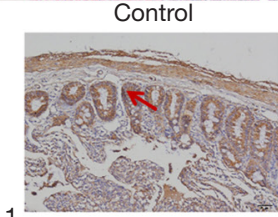

claudin-1
$(200 \times)$

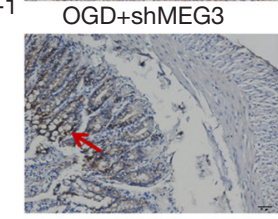

Control

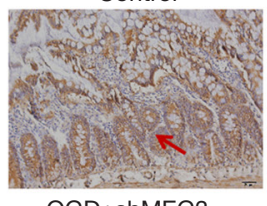

Occludi

OGD+shMEG3

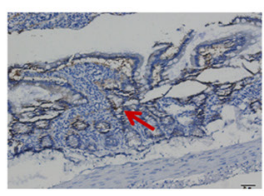

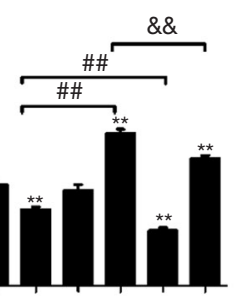

OGD

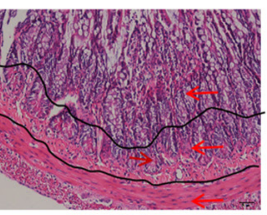

OGD

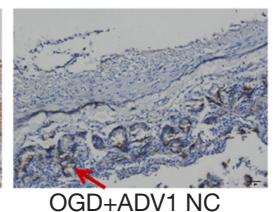

OGD+ADV1 NC

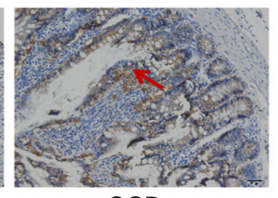

OGD

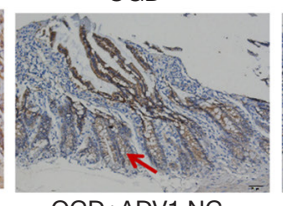

OGD+ADV1 NC

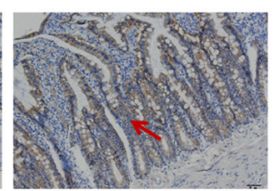

B
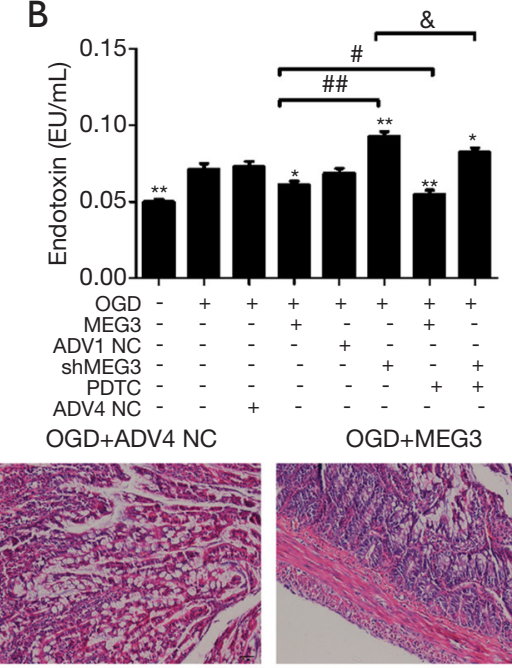

OGD+MEG3+PDTC

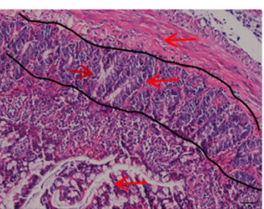

OGD+ADV4 NC

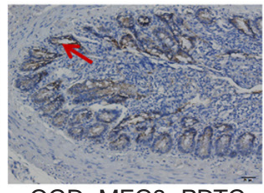

OGD+MEG3+PDTC

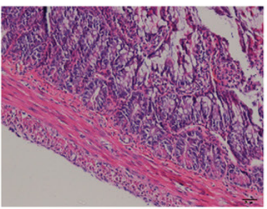

OGD+shMEG3+PDTC
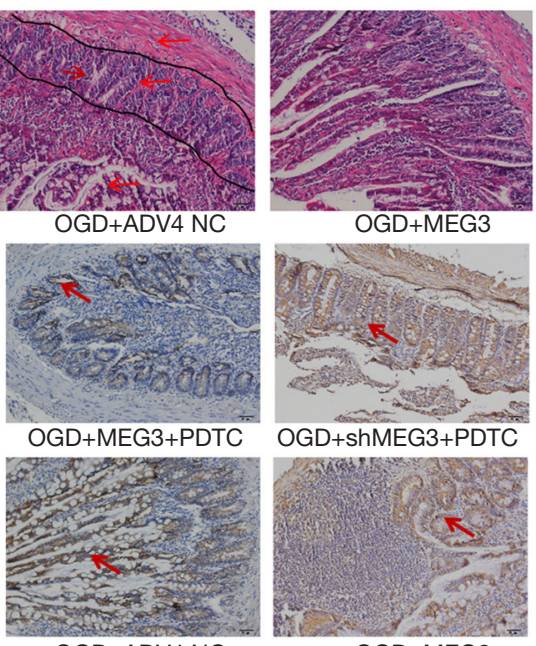

OGD+ADV4 NC

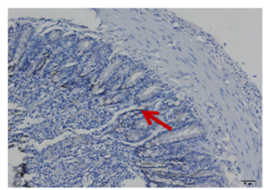

OGD+MEG3+PDTC

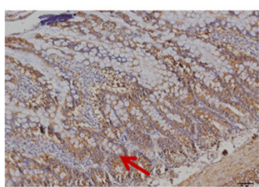

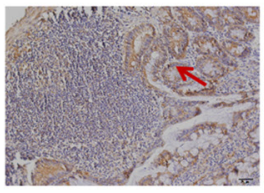

OGD+MEG3

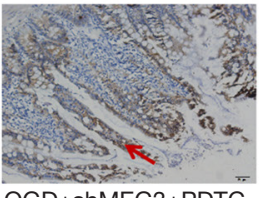

OGD+shMEG3+PDTC

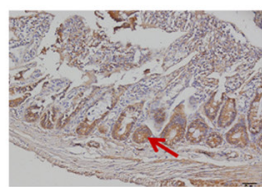

Figure 7 MEG3 relieves CA-induced intestinal barrier dysfunction through NF-кB signaling in vivo. (A-E) An in vivo CA model was established to assess the effect of CA on intestinal barrier function. Sprague-Dawley rats were intravenously injected with ADV4, ADV4 MEG3, ADV1, or ADV1 MEG3 shRNA via the tail, or treated with PTDC as indicated. (A,B) The levels of D-lactic acid and endotoxin in the serum of the rats were measured by using the corresponding test kits. (C) The intestinal injury was analyzed by hematoxylin and eosin (HE) staining in sections of intestinal mucosa from the rats. (D,E) The expression levels of occludin and claudin-1 in the intestinal mucosa of the rats were determined by immunohistochemical (IHC) staining. Mean $\pm \mathrm{SD}$ of at least three experiments is shown. Statistically significant differences are indicated: ${ }^{*} \mathrm{P}<0.05,{ }^{* *} \mathrm{P}<0.01,{ }^{\#} \mathrm{P}<0.05,{ }^{* \prime} \mathrm{P}<0.01,{ }^{\circ} \mathrm{P}<0.05,{ }^{\text {\&\&}} \mathrm{P}<0.01$. The observation positions in $(\mathrm{C}-\mathrm{E})$ are marked with red arrows. NF-kB, nuclear factor-kappa B; MEG3, maternally expressed 3; CA, Cardiac arrest. 
stress and inflammation by activating the PI3 K/Akt signaling pathway (53). G protein-coupled estrogen receptor was reported to shield the intestine from I/ $\mathrm{R}$ damage in rats by preserving the cell proliferation of crypt (54). Glial cell line-derived neurotrophic factor (GDNF) plays a vital role in the intestinal barrier in severe I/R injury (13). LncRNAs have also been found to participate in intestinal I/R injury and intestinal barrier disruption. LncRNA H19 plays a restoring role in intestinal I/R-induced intestinal barrier dysfunction (55). Repression of lncRNA NEAT1 can help to overcome the inflammatory response in inflammatory bowel disease (IBD) via modulating the intestinal barrier and through exosome-mediated macrophage polarization (56). Long noncoding RNA SPRY4-IT1 regulates intestinal barrier capacity by tempering the expression of tight junction proteins (57). In the present study, we not only demonstrated that IncRNA MEG3 protected human colorectal adenocarcinoma Caco-2 cells from OGDinduced I/R injury but also identified that MEG3 alleviated OGD-induced intestinal barrier dysfunction in vitro. Our results offer new evidence that lncRNA participates in the modulation of intestinal $\mathrm{I} / \mathrm{R}$ injury and mucosal barrier. Moreover, systemic I/R, as a primary post-cardiac arrest syndrome, is a leading cause of intestinal injury and intestinal barrier dysfunction (58). Here, we also showed that MEG3 relieved CA-induced intestinal injury and barrier dysfunction in vivo. Our study provides convincing evidence of the protective role of IncRNA in CA-related intestinal barrier.

MiRNAs have been reported to be involved in the modulation of intestinal I/R and the intestinal barrier. The dysfunction of the intestinal barrier provoked by I/R was shown to up-regulate miRNA-21 (59). Meanwhile, miRNA-378 was observed to protect against intestinal I/R insult by repressing cell apoptosis of the intestinal mucosa (60). MicroRNA-682-modulated decrease of PTEN in intestinal epithelial cells has been shown to enhance intestinal I/R injury (61). Furthermore, the repression of miR-34a-5p relieves intestinal I/R-induced apoptosis as well as the activation of oxygen species via modulating SIRT1 signaling (62). The decrease of IRAK1 by microRNA-146a can also protect the intestine against I/R injury (63).

Our study novelty reported that miR-34a-3p contributed to OGD-induced I/R injury in Caco-2 cells in vitro and is sponged by lncRNA MEG3. Our study provides valuable information that miRNA promotes intestine I/R injury. Moreover, a previous study showed that Escherichia coli nissle
1917 preserves the function of the intestinal barrier by

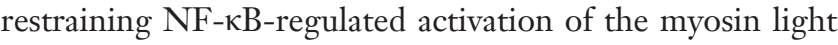
chain kinase (MLCK) signaling pathway (64). Nontoxic doses of Ochratoxin A (OTA) worsen the Deoxynivalenol $(\mathrm{DON})$-induced dysfunction of the intestinal barrier by activating the NF- $\kappa B$ pathway in IPEC-J2 cells (65). Interference of CREB-modulated zonula occludens-1 (ZO-1) and enhancement of NF- $\mathrm{KB}$-mediated IL- 6 by MCT4 impair the function of the intestinal barrier (66). Furthermore, numerous studies have shown that SIRT1 blocks the activation of NF- $\mathrm{KB}$ signaling; however, miRNA targeted to SIRT1 is able to rescue this inactivation (33-36). Moreover, SIRT1-mediated p65 inhibition is involved in I/R injury (67). SIRT1/NF- $\mathrm{kB}$ signaling plays a pivotal role in the modulation of the intestinal barrier (68). Meanwhile, Xue et al. find that down-regulation of MEG3 leads to the inhibition of inflammation and induces M2 macrophage polarization via miR-223/TRAF6/NF- $\kappa \mathrm{B}$ axis, thus alleviating VMC (69). Chen et al. reveal that MEG3 participates in caerulein-induced inflammatory injuries by targeting the miR-195-5p/FGFR2 regulatory axis via mediating the NF- $\mathrm{kB}$ pathway in HPDE cells (70). Bao et al. report that LncRNA MEG3 activates the NF- $\mathrm{BB}$ signal in GBC cells to affect the proliferation and apoptosis of GBC cells (71). Furthermore, Tong et al. find that MEG3 could alleviate HG-inducing apoptosis and inflammation via inhibiting NF- $\mathrm{KB}$ signaling pathway by targeting miR34a/SIRT1 axis (72). Those researches reveal the significant relationship between MEG3 and NF- $\kappa \mathrm{B}$ pathway. Our mechanism study showed that MEG3 inhibited the expression of inflammatory factors such as IL- $1 \beta$ and TNF- $\alpha$, as well as NF- $\kappa B$ signaling. MEG3 activates SIRT1 expression by sponging miR-34a-3p in response to the OGD treatment in vitro. These data uncover an innovative mechanism underlying the response to intestinal I/R injury.

In conclusion, we discovered that lncRNA MEG3 exerted a protective effect on intestinal barrier from CArelated I/R injury via miR-34a-3p/SIRT1/NF- $\mathrm{kB}$ signaling in vivo and in vitro. Our finding gives a new insight into the mechanism by which MEG3 restores the function of the intestinal barrier in response to $\mathrm{I} / \mathrm{R}$ injury, presenting a potential therapeutic candidate for intestinal injury.

\section{Acknowledgments}

Funding: Natural Science Foundation of Fujian Province (No.: 2020J011234); Xiamen Medical and Health Guidance Project (No. 3502720199009). 


\section{Footnote}

Reporting Checklist: The authors have completed the ARRIVE reporting checklist. Available at http://dx.doi. org/10.21037/atm-20-6438

Data Sharing Statement: Available at http://dx.doi. org/10.21037/atm-20-6438

Conflicts of Interest: All authors have completed the ICMJE uniform disclosure form (available at http://dx.doi. org/10.21037/atm-20-6438). The authors have no conflicts of interest to declare.

Ethical Statement: The authors are accountable for all aspects of the work in ensuring that questions related to the accuracy or integrity of any part of the work are appropriately investigated and resolved. Experiments were performed under a project license (NO.: 20190227) granted by the Animal Ethics Committee of the First Affiliated Hospital of Xiamen University, in compliance with the First Affiliated Hospital of Xiamen University guidelines for the care and use of animals.

Open Access Statement: This is an Open Access article distributed in accordance with the Creative Commons Attribution-NonCommercial-NoDerivs 4.0 International License (CC BY-NC-ND 4.0), which permits the noncommercial replication and distribution of the article with the strict proviso that no changes or edits are made and the original work is properly cited (including links to both the formal publication through the relevant DOI and the license). See: https://creativecommons.org/licenses/by-nc-nd/4.0/.

\section{References}

1. Jalife J. The tornadoes of sudden cardiac arrest. Nature 2018;555:597-8.

2. Lerma C, Glass L. Predicting the risk of sudden cardiac death. J Physiol 2016;594:2445-58.

3. Mitrani RD, Myerburg RJ. Ten advances defining sudden cardiac death. Trends Cardiovasc Med 2016;26:23-33.

4. Pan H, Chen D, Liu B, et al. Effects of sodium hydrosulfide on intestinal mucosal injury in a rat model of cardiac arrest and cardiopulmonary resuscitation. Life Sci 2013;93:24-9.

5. Gubernatorova EO, Liu X, Othman A, et al. EuropiumDoped Cerium Oxide Nanoparticles Limit Reactive
Oxygen Species Formation and Ameliorate Intestinal Ischemia-Reperfusion Injury. Adv Healthc Mater 2017;6.

6. Hundscheid IH, Grootjans J, Lenaerts K, et al. The Human Colon Is More Resistant to Ischemia-reperfusioninduced Tissue Damage Than the Small Intestine: An Observational Study. Ann Surg 2015;262:304-11.

7. Hoehn RS, Seitz AP, Jernigan PL, et al. Ischemia/ Reperfusion Injury Alters Sphingolipid Metabolism in the Gut. Cell Physiol Biochem 2016;39:1262-70.

8. Cauwels A, Rogge E, Vandendriessche B, et al. Extracellular ATP drives systemic inflammation, tissue damage and mortality. Cell Death Dis 2014;5:e1102.

9. Jiang LL, Zhang JJ, Zhang ZZ, et al. Effect of Intraperitoneal Resuscitation with Different Concentrations of Sodium Pyruvate on Intestinal Ischemia Reperfusion Injury in Hemorrhagic Shock Rat. Shock 2016;45:441-9.

10. Peng Z, Ban K, Wawrose RA, et al. Protection by enteral glutamine is mediated by intestinal epithelial cell peroxisome proliferator-activated receptor-gamma during intestinal ischemia/reperfusion. Shock 2015;43:327-33.

11. Camilleri M, Madsen K, Spiller R, et al. Intestinal barrier function in health and gastrointestinal disease. Neurogastroenterol Motil 2012;24:503-12.

12. Hsiao JK, Huang CY, Lu YZ, et al. Magnetic resonance imaging detects intestinal barrier dysfunction in a rat model of acute mesenteric ischemia/reperfusion injury. Invest Radiol 2009;44:329-35.

13. Xiao W, Wang W, Chen W, et al. GDNF is involved in the barrier-inducing effect of enteric glial cells on intestinal epithelial cells under acute ischemia reperfusion stimulation. Mol Neurobiol 2014;50:274-89.

14. Giuffrida P, Biancheri P, MacDonald TT. Proteases and small intestinal barrier function in health and disease. Curr Opin Gastroenterol 2014;30:147-53.

15. Chalkias A, Scheetz MH, Gulati A, et al. Periarrest intestinal bacterial translocation and resuscitation outcome. J Crit Care 2016;31:217-20.

16. Kopp F, Mendell JT. Functional Classification and Experimental Dissection of Long Noncoding RNAs. Cell 2018;172:393-407.

17. Chen J, Wan J, Ye J, et al. Emerging role of lncRNAs in the normal and diseased intestinal barrier. Inflamm Res 2018;67:757-64.

18. Wang JY, Cui YH, Xiao L, et al. Regulation of Intestinal Epithelial Barrier Function by Long Noncoding RNA uc.173 through Interaction with MicroRNA 29b. Mol Cell Biol 2018;38. 
19. Zha F, Qu X, Tang B, et al. Long non-coding RNA MEG3 promotes fibrosis and inflammatory response in diabetic nephropathy via miR-181a/Egr-1/TLR4 axis. Aging (Albany NY) 2019;11:3716-30.

20. Shi X, Sun M, Liu H, et al. Long non-coding RNAs: a new frontier in the study of human diseases. Cancer Lett 2013;339:159-66.

21. You D, You H. Repression of long non-coding RNA MEG3 restores nerve growth and alleviates neurological impairment after cerebral ischemia-reperfusion injury in a rat model. Biomed Pharmacother 2019;111:1447-57.

22. Li R, Fang L. MEG3-4 is a miRNA decoy that regulates IL-1 $\beta$ abundance to initiate and then limit inflammation to prevent sepsis during lung infection. Sci Signal 2018;11:eaao2387.

23. Piccoli MT, Gupta SK, Viereck J, et al. Inhibition of the Cardiac Fibroblast-Enriched lncRNA Meg3 Prevents Cardiac Fibrosis and Diastolic Dysfunction. Circ Res 2017;121:575-83.

24. Rupaimoole R, Slack FJ. MicroRNA therapeutics: towards a new era for the management of cancer and other diseases. Nat Rev Drug Discov 2017;16:203-22.

25. Di Leva G, Garofalo M, Croce CM. MicroRNAs in cancer. Annu Rev Pathol 2014;9:287-314.

26. Liu L, Yao J, Li Z, et al. miR-381-3p knockdown improves intestinal epithelial proliferation and barrier function after intestinal ischemia/reperfusion injury by targeting nurr1. Cell Death Dis 2018;9:411.

27. Kumar V, Mansfield J, Fan R, et al. miR-130a and miR212 Disrupt the Intestinal Epithelial Barrier through Modulation of PPARgamma and Occludin Expression in Chronic Simian Immunodeficiency Virus-Infected Rhesus Macaques. J Immunol 2018;200:2677-89.

28. Cordova-Rivas S, Fraire-Soto I, Mercado-Casas Torres A, et al. 5p and 3p Strands of miR-34 Family Members Have Differential Effects in Cell Proliferation, Migration, and Invasion in Cervical Cancer Cells. Int J Mol Sci 2019;20:545.

29. Werner TV, Hart M, Nickels R, et al. MiR-34a-3p alters proliferation and apoptosis of meningioma cells in vitro and is directly targeting SMAD4, FRAT1 and BCL2. Aging (Albany NY) 2017;9:932-54.

30. Zhai Z, Boquete JP, Lemaitre B. Cell-Specific Imd-NFkappaB Responses Enable Simultaneous Antibacterial Immunity and Intestinal Epithelial Cell Shedding upon Bacterial Infection. Immunity 2018;48:897-910.e7.

31. Meng QT, Chen R, Chen C, et al. Transcription factors Nrf2 and NF-kappaB contribute to inflammation and apoptosis induced by intestinal ischemia-reperfusion in mice. Int J Mol Med 2017;40:1731-40.

32. Turan I, Sayan Ozacmak H, Ozacmak VH, et al. The effects of S-nitrosoglutathione on intestinal ischemia reperfusion injury and acute lung injury in rats: Roles of oxidative stress and NF-kappaB. Tissue Cell 2018;52:35-41.

33. Wellman AS, Metukuri MR, Kazgan N, et al. Intestinal Epithelial Sirtuin 1 Regulates Intestinal Inflammation During Aging in Mice by Altering the Intestinal Microbiota. Gastroenterology 2017;153:772-86.

34. Chen HZ, Wang F, Gao P, et al. Age-Associated Sirtuin 1 Reduction in Vascular Smooth Muscle Links Vascular Senescence and Inflammation to Abdominal Aortic Aneurysm. Circ Res 2016;119:1076-88.

35. Zhou L, Xu DY, Sha WG, et al. High glucose induces renal tubular epithelial injury via Sirt1/NF-kappaB/ microR-29/Keap1 signal pathway. J Transl Med 2015;13:352.

36. Kong P, Yu Y, Wang L, et al. circ-Sirt1 controls NFkappaB activation via sequence-specific interaction and enhancement of SIRT1 expression by binding to miR132/212 in vascular smooth muscle cells. Nucleic Acids Res 2019;47:3580-93.

37. Zhang J, Xie X, Pan H, et al. Role of Endoplasmic Reticulum Stress in Brain Damage After Cardiopulmonary Resuscitation in Rats. Shock 2015;44:65-71.

38. Zhou W, Yao J, Wang G, et al. PKCzeta phosphorylates TRAF2 to protect against intestinal ischemia-reperfusioninduced injury. Cell Death Dis 2017;8:e2935.

39. Brentnall M, Rodriguez-Menocal L, De Guevara RL, et al. Caspase-9, caspase-3 and caspase-7 have distinct roles during intrinsic apoptosis. BMC Cell Biol 2013;14:32.

40. Zhang Y, Yang X, Ge X, et al. Puerarin attenuates neurological deficits via $\mathrm{Bcl}-2 / \mathrm{Bax} /$ cleaved caspase-3 and Sirt3/SOD2 apoptotic pathways in subarachnoid hemorrhage mice. Biomed Pharmacother 2019;109:726-33.

41. Seike S, Takehara M, Takagishi T, et al. Delta-toxin from Clostridium perfringens perturbs intestinal epithelial barrier function in Caco-2 cell monolayers. Biochim Biophys Acta Biomembr 2018;1860:428-33.

42. Fu Q, Wang H, Xia M, et al. The effect of phytic acid on tight junctions in the human intestinal Caco-2 cell line and its mechanism. Eur J Pharm Sci 2015;80:1-8.

43. Shishodia S, Majumdar S, Banerjee S, et al. Ursolic acid inhibits nuclear factor-kappaB activation induced by carcinogenic agents through suppression of IkappaBalpha kinase and p65 phosphorylation: correlation with downregulation of cyclooxygenase 2 , matrix metalloproteinase 9 , 
and cyclin D1. Cancer Res 2003;63:4375-83.

44. Ozaki I, Zhang H, Mizuta T, et al. Menatetrenone, a vitamin K2 analogue, inhibits hepatocellular carcinoma cell growth by suppressing cyclin D1 expression through inhibition of nuclear factor kappaB activation. Clin Cancer Res 2007;13:2236-45.

45. Zhang JX, Chen ZH, Chen DL, et al. LINC01410-miR532-NCF2-NF-kB feedback loop promotes gastric cancer angiogenesis and metastasis. Oncogene 2018;37:2660-75.

46. Chen Y, Qiu J, Chen B, et al. Long non-coding RNA NEAT1 plays an important role in sepsis-induced acute kidney injury by targeting miR-204 and modulating the NF-kappaB pathway. Int Immunopharmacol 2018;59:252-60.

47. Kauppinen A, Suuronen T, Ojala J, et al. Antagonistic crosstalk between NF-kappaB and SIRT1 in the regulation of inflammation and metabolic disorders. Cell Signal 2013;25:1939-48

48. Khan M, Shah SA, Kim MO. 17beta-Estradiol via SIRT1/ Acetyl-p53/NF-kB Signaling Pathway Rescued Postnatal Rat Brain Against Acute Ethanol Intoxication. Mol Neurobiol 2018;5 5:3067-78.

49. Liu Z, Li L, Chen W, et al. Aryl hydrocarbon receptor activation maintained the intestinal epithelial barrier function through Notch1 dependent signaling pathway. Int J Mol Med 2018;41:1560-72.

50. Zu G, Yao J, Ji A, et al. Nurr1 promotes intestinal regeneration after ischemia/reperfusion injury by inhibiting the expression of p21 (Waf1/Cip1). J Mol Med (Berl) 2017;95:83-95.

51. Ding C, Han F, Xiang H, et al. Probiotics ameliorate renal ischemia-reperfusion injury by modulating the phenotype of macrophages through the IL-10/GSK-3beta/PTEN signaling pathway. Pflugers Arch 2019;471:573-81.

52. Hu Q, Ren J, Li G, et al. The mitochondrially targeted antioxidant MitoQ protects the intestinal barrier by ameliorating mitochondrial DNA damage via the Nrf2/ ARE signaling pathway. Cell Death Dis 2018;9:403.

53. Chen S, Li X, Wang Y, et al. Ginsenoside Rb1 attenuates intestinal ischemia/reperfusioninduced inflammation and oxidative stress via activation of the PI3K/Akt/Nrf2 signaling pathway. Mol Med Rep 2019;19:3633-41.

54. Chai S, Liu K, Feng W, et al. Activation of G proteincoupled estrogen receptor protects intestine from ischemia/reperfusion injury in mice by protecting the crypt cell proliferation. Clin Sci (Lond) 2019;133:449-64.

55. Zou T, Jaladanki SK, Liu L, et al. H19 Long Noncoding RNA Regulates Intestinal Epithelial Barrier Function via
MicroRNA 675 by Interacting with RNA-Binding Protein HuR. Mol Cell Biol 2016;36:1332-41.

56. Liu R, Tang A, Wang X, et al. Inhibition of lncRNA NEAT1 suppresses the inflammatory response in IBD by modulating the intestinal epithelial barrier and by exosome-mediated polarization of macrophages. Int J Mol Med 2018;42:2903-13.

57. Xiao L, Rao JN, Cao S, et al. Long noncoding RNA SPRY4-IT1 regulates intestinal epithelial barrier function by modulating the expression levels of tight junction proteins. Mol Biol Cell 2016;27:617-26.

58. Binks A, Nolan JP. Post-cardiac arrest syndrome. Minerva Anestesiol 2010;76:362-8.

59. Zhang L, Zhang F, He DK, et al. MicroRNA-21 is upregulated during intestinal barrier dysfunction induced by ischemia reperfusion. Kaohsiung J Med Sci 2018;34:556-63.

60. Li Y, Wen S, Yao X, et al. MicroRNA-378 protects against intestinal ischemia/reperfusion injury via a mechanism involving the inhibition of intestinal mucosal cell apoptosis. Cell Death Dis 2017;8:e3127.

61. Liu Z, Jiang J, Yang Q, et al. MicroRNA-682-mediated downregulation of PTEN in intestinal epithelial cells ameliorates intestinal ischemia-reperfusion injury. Cell Death Dis 2016;7:e2210.

62. Wang G, Yao J, Li Z, et al. miR-34a-5p Inhibition Alleviates Intestinal Ischemia/Reperfusion-Induced Reactive Oxygen Species Accumulation and Apoptosis via Activation of SIRT1 Signaling. Antioxid Redox Signal 2016;24:961-73.

63. Chassin C, Hempel C, Stockinger S, et al. MicroRNA146a-mediated downregulation of IRAK1 protects mouse and human small intestine against ischemia/reperfusion injury. EMBO Mol Med 2012;4:1308-19.

64. Guo S, Chen S, Ma J, et al. Escherichia coli Nissle 1917 Protects Intestinal Barrier Function by Inhibiting NF-kappaB-Mediated Activation of the MLCKP-MLC Signaling Pathway. Mediators Inflamm 2019;2019:5796491.

65. Ying $\mathrm{C}$, Hong $W$, Nianhui $Z$, et al. Nontoxic concentrations of OTA aggravate DON-induced intestinal barrier dysfunction in IPEC-J2 cells via activation of NFkappaB signaling pathway. Toxicol Lett 2019;311:114-24.

66. Zhang $\mathrm{S}, \mathrm{Xu} \mathrm{W}$, Wang $\mathrm{H}$, et al. Inhibition of CREBmediated ZO-1 and activation of NF-kappaB-induced IL-6 by colonic epithelial MCT4 destroys intestinal barrier function. Cell Prolif 2019;52:e12673.

67. Liu J, Su Z, Zeng Y, et al. miR-922 regulates CYLD 
expression and promotes the cell proliferation of human hepatocellular carcinoma. Oncol Rep 2017;37:1445-50.

68. Yao B, He J, Yin X, et al. The protective effect of lithocholic acid on the intestinal epithelial barrier is mediated by the vitamin D receptor via a SIRT1/Nrf2 and NF-kappaB dependent mechanism in Caco-2 cells. Toxicol Lett 2019;316:109-18.

69. Xue YL, Zhang SX, Zheng CF, et al. Long non-coding RNA MEG3 inhibits M2 macrophage polarization by activating TRAF6 via microRNA-223 down-regulation in viral myocarditis. J Cell Mol Med 2020;24:12341-54.

70. Chen X, Song D. LncRNA MEG3 Participates in Caerulein-Induced Inflammatory Injury in Human

Cite this article as: Huang X, Pan M, Du P, Chen Y, Zhang C, Lu W, Lin J. Maternally expressed 3 protects the intestinal barrier from cardiac arrest-induced ischemia/reperfusion injury via miR-34a-3p/sirtuin 1/nuclear factor kappa B signaling. Ann Transl Med 2021;9(2):122. doi: 10.21037/atm-20-6438
Pancreatic Cells via Regulating miR-195-5p/FGFR2 Axis and Inactivating NF-кB Pathway. Inflammation 2021;44:160-73.

71. Bao D, Yuan RX, Zhang Y. Effects of lncRNA MEG3 on proliferation and apoptosis of gallbladder cancer cells through regulating NF- $\kappa \mathrm{B}$ signaling pathway. Eur Rev Med Pharmacol Sci 2020;24:6632-8.

72. Tong P, Peng QH, Gu LM, et al. LncRNA-MEG3 alleviates high glucose induced inflammation and apoptosis of retina epithelial cells via regulating miR-34a/SIRT1 axis. Exp Mol Pathol 2019;107:102-9.

(English Language Editor: B. Draper) 